\title{
MULTIGRID PRECONDITIONING OF LINEAR SYSTEMS FOR INTERIOR POINT METHODS APPLIED TO A CLASS OF BOX-CONSTRAINED OPTIMAL CONTROL PROBLEMS
}

\author{
ANDREI DRĂGĂNESCU* AND COSMIN PETRA ${ }^{\dagger}$
}

\begin{abstract}
In this article we construct and analyze multigrid preconditioners for discretizations of operators of the form $\mathcal{D}_{\lambda}+\mathcal{K}^{*} \mathcal{K}$, where $D_{\lambda}$ is the multiplication with a relatively smooth function $\lambda>0$ and $\mathcal{K}$ is a compact linear operator. These systems arise when applying interior point methods to the minimization problem $\min _{u} \frac{1}{2}\left(\|\mathcal{K} u-f\|^{2}+\beta\|u\|^{2}\right)$ with box-constraints $\underline{u} \leqslant u \leqslant \bar{u}$ on the controls. The presented preconditioning technique is closely related to the one developed by Drăgănescu and Dupont in [13] for the associated unconstrained problem, and is intended for large-scale problems. As in 13, the quality of the resulting preconditioners is shown to increase as $h \downarrow 0$, but decreases as the smoothness of $\lambda$ declines. We test this algorithm first on a Tikhonovregularized backward parabolic equation with box-constraints on the control, and then on a standard elliptic-constrained optimization problem. In both cases it is shown that the number of linear iterations per optimization step, as well as the total number of fine-scale matrix-vector multiplications is decreasing with increasing resolution, thus showing the method to be potentially very efficient for truly large-scale problems.
\end{abstract}

Key words. multigrid, interior point methods, PDE-constrained optimization

AMS subject classifications. 65M55, 90C51, 65K10,65M32, 90C06

1. Introduction. In this work we present a multigrid preconditioning technique for solving linear systems arising when applying interior point methods to the controlconstrained optimal control problem

$$
\text { minimize } \mathcal{J}_{\beta}(u) \stackrel{\text { def }}{=} \frac{1}{2}\|\mathcal{K} u-f\|^{2}+\frac{\beta}{2}\|u\|^{2}, \quad u \in \mathcal{U}_{\text {ad }},
$$

where $\Omega \subset \mathbb{R}^{d}$ is a bounded domain, $\beta>0, \mathcal{K}: L^{2}(\Omega) \rightarrow L^{2}(\Omega)$ is a linear, compact operator, and the set of admissible solutions is given by

$$
\mathcal{U}_{\mathrm{ad}}=\left\{u \in L^{2}(\Omega): \underline{u} \leqslant u \leqslant \bar{u} \text { a.e. }\right\},
$$

with $\underline{u}, \bar{u} \in L^{2}(\Omega)$, and $\underline{u} \leqslant \bar{u}$. The following examples form the main motivation for the present work:

Example A: Box-constrained time-reversal for a parabolic equation. Consider the linear parabolic initial value problem

$$
\left\{\begin{array}{lll}
\partial_{t} y+\mathcal{A} y=0 & , & \text { on } \Omega \times(0, \infty) \\
y(x, t)=0 & , & \text { on } \partial \Omega \times(0, \infty) \\
y(x, 0)=u(x), & \text { for } x \in \Omega,
\end{array}\right.
$$

where $\mathcal{A}$ is a linear elliptic operator, and denote the solution map by $\mathcal{S}(t) u \stackrel{\text { def }}{=} y(\cdot, t)$. To formulate a control problem we define $\mathcal{K}=\mathcal{S}(T)$, where $T>0$ is a fixed "endtime" $T>0$; the resulting optimization problem is controlled by the initial value $u$.

*Department of Mathematics and Statistics, University of Maryland, Baltimore County, 1000 Hilltop Circle, Baltimore, Maryland 21250 (draga@umbc.edu). The work of this author was supported in part by the Department of Energy under contract no. DE-SC0005455, and by the National Science Foundation under awards DMS-1016177 and DMS-0821311.

${ }^{\dagger}$ Mathematics and Computer Science Division, Argonne National Laboratory, 9700 S Cass Avenue, Argonne, IL 60439 (petra@mcs.anl.gov). The work of this author was supported in part by the National Science Foundation under award CCF-0728878. 
Note that if the box constraints in (1.1) are left out, i.e., $\mathcal{U}_{\mathrm{ad}}=L^{2}(\Omega)$, then (1.1) is the Tikhonov-regularized formulation of the ill-posed problem

$$
\mathcal{K} u=f .
$$

\section{Example B: Elliptic-constrained distributed optimal control problem.}

In this example we let

$$
\mathcal{K}=\Delta^{-1},
$$

where $\Delta$ is the Laplace operator acting on $H_{0}^{1}(\Omega)$. In this case the problem (1.1) is usually formulated as the PDE-constrained optimization problem (e.g., see Borzi and Schulz [8])

$$
\begin{aligned}
& \text { minimize } \\
& \text { subj. to } \quad \Delta y=u, y \in H_{0}^{1}(\Omega), \underline{u} \leqslant u \leqslant \bar{u} \quad \text { a.e. in } \Omega .
\end{aligned}
$$

Our primary motivation is rooted in solving large-scale inverse problems like the one in Example A, which is a simplified version of the problem considered by Akçelik et al. in 2. There the question was to identify the initial concentration $u=y(\cdot, 0)$ of a contaminant released in the atmosphere in a given geographic area (the Los Angeles Basin) given later-time measurements at various fixed locations. The spatio-temporal evolution of the concentration $y$ of the contaminant is assumed, like in Example A, to be governed by an advection-diffusion equation with known wind-velocities and contaminant diffusivity that can be formulated as (1.2). In Example A we consider the case where the measurements are taken at all points in space but at a single moment in time $T>0$, therefore the data $f$ is the entire state at time $T$. The problem thus becomes to invert a compact operator which, as is well known, is not continuously invertible. As a result, a naive approach to inverting $\mathcal{K}$ is unstable, in that small perturbations in the "measurements" $f$ result in exponentially large errors in the solution. If the "exact" measurements $f$ are resulted from applying $\mathcal{K}$ to a "true" initial value $u$, that is, $f=\mathcal{K} u$, then various regularization techniques [15] are employed so that the computed solution $u_{\delta}$ of the $\delta$-perturbed problem $\mathcal{K} u_{\delta}=f_{\delta}$ (where $\left\|f-f_{\delta}\right\| \leqslant \delta$ ) converges to the "true" solution $u$ as $\delta \downarrow 0$, the most commonly used being the Tikhonov-regularization. One issue not resolved by the Tikhonov regularization is that the solution $u_{\delta}$ of the regularized problem may exhibit non-physical, or otherwise qualitatively incorrect behavior: for example, if the concentration needs to have values in $[0,1]$, it is well known that $u_{\delta}$ may exceed these limits. In addition, if the true initial contamination event is localized, then $u_{\delta}$ oscillates around zero, and is not localized. However, if explicit constraints $\underline{u}=0, \bar{u}=1$ are set, then the solution is physically relevant, and is also localized. In Figure 1.1 we show the solution of the inverse problem where the target solution (true initial concentration) is represented by two localized sources of contamination, with one dominating the other in size. The solution of the unconstrained problem (with $\delta$ down to roundoff error and $\beta$ optimized) does not show two sources of pollution, unlike the solution of the constrained problem, from which one can clearly identify two separate components in the support of the initial value. In addition, in the presence of nonlinearities, with reaction terms that are sensitive to the sign of their arguments, the importance of physically meaningful box constraints cannot be overstated. A similar situation is encountered in image-deblurring, where the target (gray-scale) solution takes values in $[0,1]$; if 


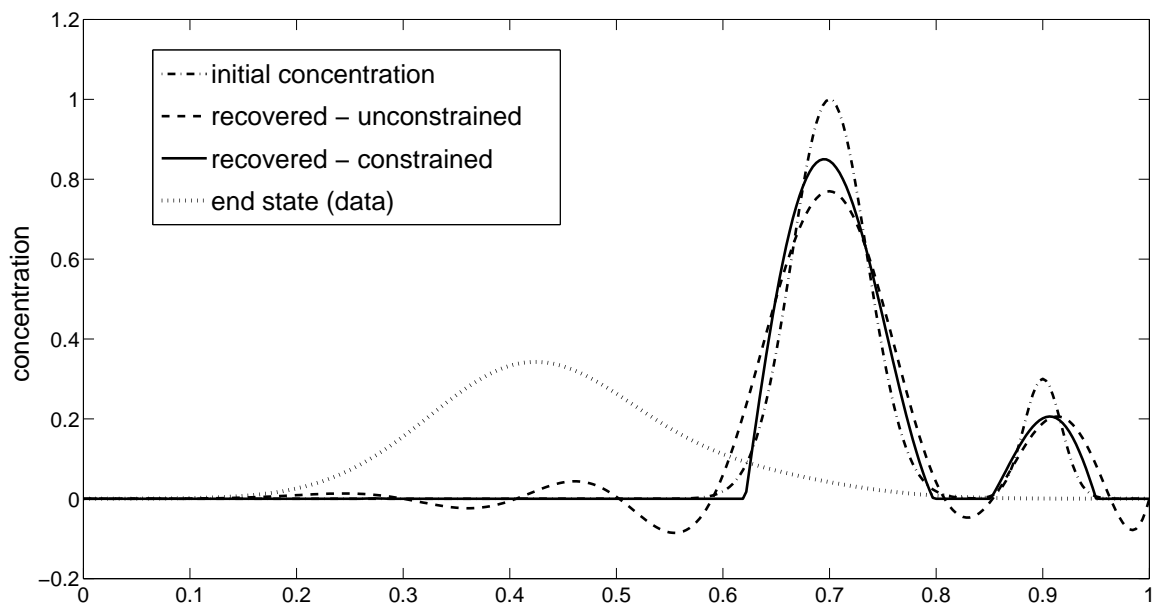

FIG. 1.1. Solution of the constrained vs. unconstrained inverse problem, with the target solution (initial concentration) being a smooth function supported on two disjoint intervals.

these boundaries are strictly enforced, then the quality of inversion (deblurring) is significantly improved 34 .

The unconstrained optimization problem, where $\mathcal{U}_{\mathrm{ad}}=L^{2}(\Omega)$ in (1.1), ultimately reduces to solving the normal equations

$$
\left(\beta I+\mathcal{K}^{*} \mathcal{K}\right) u=\mathcal{K}^{*} f .
$$

For compact operators these systems either represent or resemble very well integral equations of the second kind. Starting with the works of Hackbusch [20] (see also [22]) much effort has been devoted to developing efficient multigrid methods for solving (1.5), e.g., see 28, 33, 23, 27, 5, 13, and the references therein. We should point out that multigrid methods were originally developed for solving elliptic equations [21, 10, and later significant efforts were devoted to extending these methods to other important differential equations such as advection-diffusion and the NavierStokes equations (e.g., see 14 and the references therein). For elliptic equations, typically the goal is to reduce the condition number of the discrete system from $O\left(h^{-2}\right)$ to $O(1)$, which results in a solution process that solves the equation in a number of iterations that is mesh-independent. However, the discrete version of (1.5) has a condition number which is $O\left(\beta^{-1}\right)$, with the bound being independent of $h$. Moreover, even for $\beta=0$, conjugate gradient (used as a regularizer) already solves (1.5) in a mesh-independent number of iterations [15]; in other words, mesh-independence is nearly effortless for integral equations. Instead, for systems like (1.5) multigrid is used to further reduce the condition number of the preconditioned system. For example, in 13 it is shown that by using specially designed multigrid preconditioners, the preconditioned version of (1.5) has a condition number bounded by $O\left(h^{2} / \beta\right)$, the consequence of which is interesting at least from a theoretical point of view: if $\beta>0$ is kept fixed (which is normally not the case in practical applications) the number of iterations required to solve the problem decreases with $h \downarrow 0$ to the point where only one iteration would be enough to solve the problem with sufficient 
accuracy. This fact, already known to Hackbusch [20, constitutes a departure from the usual multigrid framework, where, as stated before, the goal is to achieve meshindependence (bounded number of iterations as $h \downarrow 0$ ). Hence, as a result of specific multigrid preconditioning, the solution process for the unconstrained problem (1.5) requires fewer and fewer fine-scale matrix-vector multiplications (mat-vecs) as $h \downarrow 0$. The main contribution of the present work is to show that such performance can also be achieved in the presence of explicit box constraints on the control, as formulated in (1.1).

Multigrid methods have long been associated with solving large-scale problems, and beginning with the work of Hackbusch [18, 19, and especially over the last decade, significant efforts were concentrated on devising efficient multilevel methods for optimal control problems $3,3,6,6,40,29,4$. A more detailed discussion of the subject and many references can be found in the recent review article by Borzi and Schulz [8]. While most - not all - of the aforementioned articles discuss unconstrained problems, the addition of box constraints pose additional challenges associated with the presence of the Lagrange multipliers related to the inequality-constraints, which in general are less regular than the solutions. Optimization methods for such problems with bound constraints typically fall in one of two categories: active-set type strategies, especially semismooth Newton methods (SSNMs) and interior point methods (IPMs). Over the last decade both IPMs and SSNMs have consistently attracted the attention of the scientific community due to their proven efficiency in solving distributed optimal control problems with PDE constraints. Both strategies lead to superlinear local convergence [38, 39, 24] and lend themselves to analysis both in a finite dimensional setting and in function space [37, 41] (see also [26]), which is a critical stepping stone to proving mesh-independence [25]. Each of IPMs and SSNMs consists of an outer iterative process that further requires solving one or two linear systems at each outer iteration. For large-scale problems the solution of these linear systems often becomes the bottleneck of the computation. In terms of their linear algebra needs, IPMs and SSNMs exhibit significant differences and require separate treatment. For SSNMs, the linear systems involve a subset of unknowns and equations of the Hessian of the cost functional, while for IPMs the systems have the same structure as the systems arising in the unconstrained problem, but contain additional terms on the diagonal which usually are a source of extreme ill-conditioning. The question of efficient multigrid preconditioning of the linear systems arising in the semismooth Newton solution process is the subject of current research [11].

The focus of the current work addresses the question of efficient multigrid preconditioning of linear systems arising in the IPM solution process. Multigrid and IPMs have been shown to work well together for elliptic variational inequalities (obstacle problems) [3, and for some classes of problems where the Hessian of the cost functional is elliptic [4. Instead, the Hessian of the cost functional in (1.1) is a compact operator and requires a significantly different approach.

In this article we treat $\mathcal{K}$ and its discretizations as black-box operators, and we employ the first-discretize-then-optimize strategy. We apply specific primal-dual IPMs to the discrete version of (1.1), and we develop multigrid preconditioners for the linear systems arising at each IPM iterate. As shown in Section 3 if the discrete optimal control problem is formulated appropriately, then the linear systems to be solved involve matrices of the form $\mathbf{D}+\mathbf{K}^{*} \mathbf{K}$, where $\mathbf{D}$ is a diagonal matrix, $\mathbf{K}$ is the discrete representation of $\mathcal{K}$, and $\mathbf{K}^{*}$ is the adjoint of $\mathbf{K}$ with respect to a certain discrete inner product. When using standard finite elements, the matrices $\mathbf{K}, \mathbf{K}^{*}$ 
are dense; consequently, for large-scale problems, they cannot be formed and the systems are solved using iterative methods. Since residuals can be computed at the equivalent cost of two applications of $\mathbf{K}$, residual computation is expected to be very expensive, therefore efficient preconditioners are critical for minimizing the number of necessary mat-vecs. Our strategy in this work is to adapt the multigrid techniques developed in [13, where the matrix $\mathbf{D}$ had the form $\beta \mathbf{I}$. To analyse the resulting multigrid preconditioner we interpret $\mathbf{D}$ as being the discretization of an operator $\mathcal{D}_{\lambda}$, where $\left(\mathcal{D}_{\lambda} u\right)(x)=\lambda(x) u(x)$ is the operator representing pointwise multiplication of a function $u$ with a smooth function 1 . From a technical perspective, our main accomplishment consists of showing that the operators of the type $\mathcal{K} \mathcal{D}_{\lambda}$ together with their discretizations satisfy a set of smoothing conditions shown in [13] to be sufficient for the multigrid preconditioner to have the desired qualities.

For simplicity and concreteness we restrict most of our study to the two-dimensional case, and we consider a standard finite element discretization for $\mathcal{K}$ using triangular elements and continuous piecewise linear functions. As will result from the analysis, these techniques can be easily generalized to three dimensions and rectangular elements, however, the extension to higher degree finite elements is not obvious and forms the subject of current research.

This article is organized as follows: After formally introducing the discrete optimization problem in Section 2, we discuss the specific linear algebra requirements of the interior point methods in Section 3. Section 4 is central to this work as it presents the analysis of the two-grid preconditioner, the main result being Theorem 4.9, In Section 5 we develop a multigrid preconditioner that preserves the qualities of the two-grid preconditioner. Further, we apply the methods to Examples A and B in Section 6 and show some numerical results.

2. Notation and discrete problem formulation. Throughout this paper we shall denote by $W_{p}^{m}(\Omega), H^{m}(\Omega), H_{0}^{m}(\Omega)$ (with $p \in[1, \infty], m \in \mathbb{N}$ ) the standard Sobolev spaces, while $\|\cdot\|$ and $\langle\cdot, \cdot\rangle$ are the $L^{2}$-norm and inner product, respectively. Let $\widetilde{H}^{-m}(\Omega)$ be the dual (with respect to the $L^{2}$-inner product) of $H^{m}(\Omega) \cap H_{0}^{1}(\Omega)$ for $m>0$. If $X$ is a Banach space then $\mathfrak{L}(X)$ denotes the space of bounded linear operators on $X$. We regard square $n \times n$ matrices as operators in $\mathfrak{L}\left(\mathbb{R}^{n}\right)$ and we write matrices and vectors using bold font. If $\mathbf{A}$ is a symmetric positive definite matrix, we denote by $(\mathbf{u}, \mathbf{v})_{\mathbf{A}}=\mathbf{v}^{T} \mathbf{A u}$ the $\mathbf{A}$-dot product of two vectors $\mathbf{u}, \mathbf{v}$, and by $|\mathbf{u}|_{\mathbf{A}}=\sqrt{(\mathbf{u}, \mathbf{u})_{\mathbf{A}}}$ the $\mathbf{A}$-norm; if $\mathbf{A}=\mathbf{I}$ we drop the subscript from the inner product and norm. The space of $m \times n$ matrices is denoted by $M_{m \times n}$; if $m=n$ we write $M_{n}$ instead of $M_{m \times n}$. Given some norm $\|\cdot\|_{s}$ on a vector space $\mathcal{X}$, and $T \in \mathfrak{L}(\mathcal{X})$, we denote by $\|T\|_{s}$ the induced operator-norm

$$
\|T\|_{s}=\sup _{u \in \mathcal{X},\|u\|_{s}=1}\|T u\|_{s} .
$$

Consequently, if $T \in \mathfrak{L}\left(L^{2}(\Omega)\right)$ then $\|T\|$ (no subscripts) is the $L^{2}$ operator-norm of $T$. If $\mathcal{X}$ is a Hilbert space and $T \in \mathfrak{L}(\mathcal{X})$ then $T^{*} \in \mathfrak{L}(\mathcal{X})$ denotes the adjoint of $T$.

We assume that $\Omega \subset \mathbb{R}^{2}$ is a bounded, polygonal domain and that $\mathcal{K}$ is discretized using continuous piecewise linear functions on triangular elements. We consider the usual multigrid framework where the operator is discretized at several resolutions. Let $\mathcal{T}_{h_{0}}$ be a triangulation of the domain $\Omega$, and define $\mathcal{T}_{h / 2}$ inductively to be the

\footnotetext{
${ }^{1}$ This can always be accomplished, $\lambda$ can be any smooth (here $C^{2}$ is sufficient) interpolant of the discrete function representing the diagonal of $\mathbf{D}$.
} 
Goursat refinement of $\mathcal{T}_{h}$ for all $h \in I$ with

$$
I=\left\{h_{0} / 2^{i}: i=0,1,2, \ldots\right\},
$$

where each triangle in $T \in \mathcal{T}_{h}$ is cut along the three lines obtained by joining the midpoints of its edges. Note that $\left(\mathcal{T}_{h}\right)_{h \in I}$ is a quasi-uniform triangulation and the usual approximations hold [9]. We define

$$
\mathcal{V}_{h}=\left\{u \in \mathcal{C}(\bar{\Omega}):\left.\forall T \in \mathcal{T}_{h} \quad u\right|_{T} \text { is linear, and }\left.u\right|_{\partial \Omega} \equiv 0\right\},
$$

so that $\mathcal{V}_{h / 2} \subset \mathcal{V}_{h} \subset H_{0}^{1}(\Omega)$. We should note that zero-boundary conditions for the controls are consistent with the examples considered, and present a convenient framework for the analysis, but alternate boundary conditions can be considered. For Example A, setting $u$ to be zero on $\partial \Omega$ is natural, while in Example B the boundary values of the control do not enter the discrete problem unless higher order cubatures are used in the discretization. If $N_{h}=\operatorname{dim}\left(\mathcal{V}_{h}\right)$ and $P_{1}^{h}, \ldots, P_{N_{h}}^{h}$ are the nodes of $\mathcal{T}_{h}$ that lie in the interior of $\Omega$ let $\mathcal{I}_{h}: \mathcal{C}(\Omega) \rightarrow \mathcal{V}_{h}$ be the standard interpolation operator

$$
\mathcal{I}_{h}(u)=\sum_{i=1}^{N_{h}} u\left(P_{i}^{h}\right) \varphi_{i}^{h},
$$

where $\varphi_{i}^{h}, i=1, \ldots, N_{h}$ are the standard nodal basis functions. Given a family of positive weight-functions $\left(w_{h}\right)_{h \in I} \subset \mathcal{V}_{h}$ we define the mesh-dependent inner products

$$
\langle u, v\rangle_{h}=\sum_{i=1}^{N_{h}} w_{h}\left(P_{i}^{h}\right) u\left(P_{i}^{h}\right) v\left(P_{i}^{h}\right), \quad \text { for } u, v \in \mathcal{V}_{h},
$$

and let $\|u\|_{h}=\sqrt{\langle u, u\rangle_{h}}$. In order to satisfy $\langle\cdot, \cdot\rangle_{h} \approx\langle\cdot, \cdot\rangle$ as close as possible we replace exact integration on each triangle $\Delta P_{1} P_{2} P_{3}$ with the cubature

$$
\int_{T} f(x) d x \approx Q(f)=\frac{\operatorname{area}(T)}{3} \sum_{i=1}^{3} f\left(P_{i}\right) .
$$

This defines the weight functions $w_{h}$

$$
w_{h}\left(P_{i}^{h}\right)=\frac{1}{3} \sum_{P_{i}^{h} \in T \in \mathcal{T}_{h}} \operatorname{area}(T) .
$$

Since the cubature $Q$ is exact for linear functions [35] we have

$$
\langle u, v\rangle_{h}=\int_{\Omega} \mathcal{I}_{h}(u v), \text { for all } u, v \in \mathcal{V}_{h} .
$$

Moreover, if the grids are quasi-uniform, then $h^{-2} w_{h}$ are uniformly bounded and bounded away from 0 with respect to $h \in I$, therefore by Lemma 6.2.7 in [9] there exist positive constants $C_{1}, C_{2}$ independent of $h$ such that

$$
C_{1}\|u\| \leqslant\|u\|_{h} \leqslant C_{2}\|u\|, \forall u \in \mathcal{V}_{h} .
$$

We should point out that the norm-equivalence (2.2) extends to operator norms for operators in $\mathfrak{L}\left(L^{2}(\Omega)\right)$, which allows us to interchange $\|T\|$ with $\|T\|_{h}$ when needed as long as we factor in a mesh-independent constant. 
We assume that for each $h \in I$ is given a natural discretization $\mathcal{K}_{h} \in \mathfrak{L}\left(\mathcal{V}_{h}\right)$ of $\mathcal{K}$, so that $\mathcal{K}, \mathcal{K}_{h}$ satisfy the Smoothed Approximation Condition (SAC):

Condition 2.1 (SAC). An operator $\mathcal{M}$ together with its discretization $\mathcal{M}_{h}$ is said to satisfy the Smoothed Approximation Condition if there exists a constant $C(\mathcal{M})$ depending on $\mathcal{M}, \Omega, \mathcal{T}_{h_{0}}$ and independent of $h$ so that

[a] smoothing:

$$
\|\mathcal{M} u\|_{H^{m}(\Omega)} \leqslant C(\mathcal{M})\|u\|, \quad \forall u \in L^{2}(\Omega), m=0,1,2 ;
$$

[b] smoothed approximation:

$$
\left\|\mathcal{M} u-\mathcal{M}_{h} u\right\|_{H^{m}(\Omega)} \leqslant C(\mathcal{M}) h^{2-m}\|u\| \quad \forall u \in \mathcal{V}_{h}, m=0,1, h \in I .
$$

Given two discrete functions $\underline{u}_{h}, \bar{u}_{h} \in \mathcal{V}_{h}$ representing $\underline{u}, \bar{u}$, respectively, we now define the discrete optimization problem

$$
\text { minimize } \mathcal{J}_{\beta}^{h}(u) \stackrel{\text { def }}{=} \frac{1}{2}\left\|\mathcal{K}_{h} u-f_{h}\right\|_{h}^{2}+\frac{\beta}{2}\|u\|_{h}^{2}, \quad u \in \mathcal{U}_{\mathrm{ad}}^{h},
$$

where $f_{h} \in \mathcal{V}_{h}$ represents $f$ and the set of discrete admissible solutions is given by

$$
\mathcal{U}_{\mathrm{ad}}^{h}=\left\{u \in \mathcal{V}_{h}: \underline{u}_{h}\left(P_{i}^{h}\right) \leqslant u\left(P_{i}^{h}\right) \leqslant \bar{u}\left(P_{i}^{h}\right) \quad \forall i=1, \ldots, N_{h}\right\} .
$$

The formulation (2.5) needs a few comments. First we remark that the use of the discrete norm $\|\cdot\|_{h}$ instead of $\|\cdot\|$ is essential in order for the linear systems to be solved at each outer iteration to have a form amenable to efficient multigrid preconditioning. In other words, we have chosen a discretization that allows for an efficient solution process. Second, if $\underline{u}$ and $-\bar{u}$ are convex and continuous (e.g., when they are constant), then the choice

$$
\underline{u}_{h}=\mathcal{I}_{h}(\underline{u}), \quad \bar{u}_{h}=\mathcal{I}_{h}(\bar{u})
$$

implies $\mathcal{U}_{\text {ad }}^{h} \subset \mathcal{U}_{\text {ad }}$. This construction can be easily generalized to three dimensions and/or tensor-product finite elements.

We obtain a matrix formulation of (2.5) by representing all vectors and operators using the standard nodal basis functions $\varphi_{i}^{h}, i=1, \ldots, N_{h}$. More precisely, if we define $T: \mathbb{R}^{N_{h}} \rightarrow \mathcal{V}_{h}$ by

$$
T(\mathbf{u})=\sum_{i=1}^{N_{h}} u_{i} \varphi_{i}^{h}, \quad \text { where } \mathbf{u}=\left[u_{1}, \ldots, u_{N_{h}}\right]^{T},
$$

then the matrix $\mathbf{K}_{h}=T^{-1} \mathcal{K}_{h} T$, regarded as an operator in $\mathfrak{L}\left(\mathbb{R}^{N_{h}}\right)$, represents $\mathcal{K}_{h}$ with respect to the nodal basis. If $\mathbf{W}_{h}$ is the diagonal matrix with diagonal entries $\left(w_{h}\left(P_{i}^{h}\right)\right)_{1 \leqslant i \leqslant N_{h}}$, and $\mathbf{f}_{h}, \underline{\mathbf{u}}_{h}, \overline{\mathbf{u}}_{h}$ represent $f_{h}, \underline{u}_{h}, \bar{u}_{h}$ respectively, then (2.5) is equivalent to

$$
\operatorname{minimize} \quad J_{\beta}(\mathbf{u}) \stackrel{\text { def }}{=} \frac{1}{2}\left|\mathbf{K}_{h} \mathbf{u}-\mathbf{f}_{h}\right|_{\mathbf{W}_{h}}^{2}+\frac{\beta}{2}|\mathbf{u}|_{\mathbf{W}_{h}}^{2}, \quad \underline{\mathbf{u}}_{h} \leqslant \mathbf{u} \leqslant \overline{\mathbf{u}}_{h},
$$

where the inequality $\mathbf{u} \leqslant \mathbf{v}$ between vectors is meant coordinate-wise. When operating on a single grid we will omit the subscript $h$ for matrices and vectors.

Existence and uniqueness of solutions for both (1.1) and (2.5) follows from the fact that $\mathcal{J}_{\beta}, \mathcal{J}_{\beta}^{h}$ are uniformly convex and $\mathcal{U}_{\text {ad }}, \mathcal{U}_{\text {ad }}^{h}$ are convex sets (e.g., see Theorem 1.43 
in [26]). Furthermore, cf. Lemma 1.12 in [26, the solution $\widehat{u}$ of (1.1) is characterized by the following condition: there exist $\underline{\lambda}, \bar{\lambda} \in L^{2}(\Omega)$ so that

$$
\left\{\begin{array}{l}
\left(\beta I+\mathcal{K}^{*} \mathcal{K}\right) \widehat{u}+\bar{\lambda}-\underline{\lambda}=\mathcal{K}^{*} f, \\
\widehat{u} \geqslant \underline{u}, \quad \underline{\lambda} \geqslant 0, \quad \underline{\lambda}(\widehat{u}-\underline{u})=0 \quad \text { a.e. } \\
\widehat{u} \leqslant \bar{u}, \quad \bar{\lambda} \geqslant 0, \quad \bar{\lambda}(\bar{u}-\widehat{u})=0 \quad \text { a.e. }
\end{array}\right.
$$

3. Interior point methods and linear systems. In this section we briefly discuss the specifics of the interior point method we use for solving the discrete optimization problem (2.7), and we describe in detail the linear systems that need to be solved at each outer iteration. If we denote

$$
\mathbf{A} \stackrel{\text { def }}{=} \beta \mathbf{W}+\mathbf{K}^{T} \mathbf{W K},
$$

then after a rearrangement of the terms in the objective function $J_{\beta}$ and dropping constant terms we write (2.7) as a regular convex quadratic problem with affine constraints in standard form:

$$
\begin{array}{ll}
\operatorname{minimize} & \frac{1}{2} \mathbf{u}^{T} \mathbf{A} \mathbf{u}-\left(\mathbf{K}^{T} \mathbf{W} \mathbf{f}\right)^{T} \mathbf{u} \\
\text { subj to: } & \underline{\mathbf{u}} \leqslant \mathbf{u} \leqslant \overline{\mathbf{u}} .
\end{array}
$$

Since $\mathbf{W}$ is a diagonal matrix with positive entries, the matrix $\mathbf{A}$ is positive definite, therefore the above problem has a unique solution (see [32, p. 320). Let us denote the Lagrangian corresponding to the QP (3.2) by

$$
L\left(\mathbf{u}, \mathbf{v}_{1}, \mathbf{v}_{2}\right)=\frac{1}{2} \mathbf{u}^{T} \mathbf{A} \mathbf{u}-\left(\mathbf{K}^{T} \mathbf{W} \mathbf{f}\right)^{T} \mathbf{u}-\mathbf{v}_{2}^{T}(\overline{\mathbf{u}}-\mathbf{u})-\mathbf{v}_{1}^{T}(\mathbf{u}-\underline{\mathbf{u}}),
$$

where $\mathbf{v}_{1}, \mathbf{v}_{2}$ are vectors of non-negative multipliers corresponding to the inequality constraints. Then the gradient and Hessian of the Lagrangian are given by

$$
\nabla L_{\mathbf{u}}\left(\mathbf{u}, \mathbf{v}_{1}, \mathbf{v}_{2}\right)=\mathbf{A u}-\mathbf{K}^{T} \mathbf{W} \mathbf{f}+\mathbf{v}_{2}-\mathbf{v}_{1}, \quad \nabla^{2} L_{\mathbf{u u}}(\mathbf{u}, \mathbf{v})=\mathbf{A} .
$$

Since the the linear independence constraint qualification holds, the unique solution $\widehat{\mathbf{u}}$ of (3.2) satisfies the Karush-Kuhn-Tucker (KKT) conditions

$$
\left\{\begin{array}{l}
\mathbf{A} \widehat{\mathbf{u}}+\widehat{\mathbf{v}}_{2}-\widehat{\mathbf{v}}_{1}=\mathbf{K}^{T} \mathbf{W} \mathbf{f} \\
\widehat{\mathbf{u}} \geqslant \underline{\mathbf{u}}, \quad \widehat{\mathbf{v}}_{1} \geqslant 0, \quad \widehat{\mathbf{v}}_{1} \cdot(\widehat{\mathbf{u}}-\underline{\mathbf{u}})=0 \\
\widehat{\mathbf{u}} \leqslant \overline{\mathbf{u}}, \quad \widehat{\mathbf{v}}_{2} \geqslant 0, \quad \widehat{\mathbf{v}}_{2} \cdot(\overline{\mathbf{u}}-\widehat{\mathbf{u}})=0
\end{array}\right.
$$

where $\widehat{\mathbf{v}}_{1}, \widehat{\mathbf{v}}_{2}$ are the multipliers, and $\mathbf{u} \cdot \mathbf{s}$ denotes the component-wise product. Moreover, since $\nabla^{2} L_{\mathbf{u u}}$ is positive definite, the above KKT conditions are also sufficient. The primal-dual IPM consists of solving the perturbed KKT system

$$
\left\{\begin{array}{l}
\mathbf{A u}+\mathbf{v}_{2}-\mathbf{v}_{1}=\mathbf{K}^{T} \mathbf{W} \mathbf{f}, \\
\mathbf{u}>\underline{\mathbf{u}}, \quad \mathbf{v}_{1}>0, \quad \mathbf{v}_{1} \cdot(\mathbf{u}-\underline{\mathbf{u}})=\mu \mathbf{e}, \\
\mathbf{u}<\overline{\mathbf{u}}, \quad \mathbf{v}_{2}>0, \quad \mathbf{v}_{2} \cdot(\overline{\mathbf{u}}-\mathbf{u})=\mu \mathbf{e},
\end{array}\right.
$$

whose one-parameter family of solutions $\left(\widehat{\mathbf{u}}(\mu), \widehat{\mathbf{v}}_{1}(\mu), \widehat{\mathbf{v}}_{2}(\mu)\right)$ defines the central path. As usual, $\mathbf{e}=[1,1, \ldots, 1]^{T} \in \mathbb{R}^{N_{h}}$. Practical IPM algorithms produce solutions that 
lie sufficiently close to the central path and converge rapidly to $\left(\widehat{\mathbf{u}}, \widehat{\mathbf{v}}_{1}, \widehat{\mathbf{v}}_{2}\right)$. An example of such method is Mehrotra's predictor-corrector algorithm. Initially introduced for linear programming [31, the method was successfully adapted to convex QPs and emerged in the last fifteen years as the (arguably) most practical and efficient algorithm for this class of problems. For this project we used Matlab to implement Mehrotra's method for convex QPs from OOQP (see [16] for details).

To describe the method we first consider the the linear system defining the Newton direction $\left(\delta \mathbf{u}, \delta \mathbf{v}_{1}, \delta \mathbf{v}_{2}\right)$ for (3.4):

$$
\left\{\begin{aligned}
\mathbf{A} \delta \mathbf{u}+\delta \mathbf{v}_{2}-\delta \mathbf{v}_{1} & =\mathbf{K}^{T} \mathbf{W} \mathbf{f}-\mathbf{A u}-\mathbf{v}_{2}+\mathbf{v}_{1} \\
\mathbf{V}_{1} \delta \mathbf{u}+(\mathbf{U}-\underline{\mathbf{U}}) \delta \mathbf{v}_{1} & =\mu \mathbf{e}-\mathbf{v}_{1} \cdot(\mathbf{u}-\underline{\mathbf{u}}) \\
-\mathbf{V}_{2} \delta \mathbf{u}+(\overline{\mathbf{U}}-\mathbf{U}) \delta \mathbf{v}_{2} & =\mu \mathbf{e}-\mathbf{v}_{2} \cdot(\overline{\mathbf{u}}-\mathbf{u})
\end{aligned}\right.
$$

where $\mathbf{U}, \underline{\mathbf{U}}, \overline{\mathbf{U}}, \mathbf{V}_{1}$, and $\mathbf{V}_{2}$ are diagonal matrices with the diagonal given by the vectors $\mathbf{u}, \underline{\mathbf{u}}, \overline{\mathbf{u}}, \mathbf{v}_{1}$, and $\mathbf{v}_{2}$, respectively. In Mehrotra's algorithm, given the current iterate $\left(\mathbf{u}, \mathbf{v}_{1}, \mathbf{v}_{2}\right)$, one first computes the predictor direction $\left(\delta \mathbf{u}^{a}, \delta \mathbf{v}_{1}^{a}, \delta \mathbf{v}_{2}^{a}\right)$ as the solution of (3.5) with $\mu=0$. Secondly, the corrector direction $\left(\delta \mathbf{u}, \delta \mathbf{v}_{1}, \delta \mathbf{v}_{2}\right)$ is the solution of a linear system that differs from (3.5) only in the right-hand side, namely:

$$
\left\{\begin{aligned}
\mathbf{A} \delta \mathbf{u}+\delta \mathbf{v}_{2}-\delta \mathbf{v}_{1} & =\mathbf{K}^{T} \mathbf{W} \mathbf{f}-\mathbf{A u}-\mathbf{v}_{2}+\mathbf{v}_{1} \\
\mathbf{V}_{1} \delta \mathbf{u}+(\mathbf{U}-\underline{\mathbf{U}}) \delta \mathbf{v}_{1} & =\sigma \mu \mathbf{e}-\mathbf{v}_{1} \cdot(\mathbf{u}-\underline{\mathbf{u}})+\delta \mathbf{u}^{a} \cdot \delta \mathbf{v}_{1}^{a} \\
-\mathbf{V}_{2} \delta \mathbf{u}+(\overline{\mathbf{U}}-\mathbf{U}) \delta \mathbf{v}_{2} & =\sigma \mu \mathbf{e}-\mathbf{v}_{2} \cdot(\overline{\mathbf{u}}-\mathbf{u})+\delta \mathbf{u}^{a} \cdot \delta \mathbf{v}_{2}^{a}
\end{aligned}\right.
$$

where $\mu=\left((\mathbf{u}-\underline{\mathbf{u}})^{T} \mathbf{v}_{1}+(\overline{\mathbf{u}}-\mathbf{u})^{T} \mathbf{v}_{2}\right) /\left(2 N_{h}\right)$, and $\sigma>0$ is a centering parameter that is computed accordingly to Mehrotra's heuristic. Therefore, both the predictor and the corrector step involve a system - the augmented system - of the form

$$
\left[\begin{array}{ccc}
\mathbf{A} & -\mathbf{I} & \mathbf{I} \\
\mathbf{V}_{1} & (\mathbf{U}-\underline{\mathbf{U}}) & \mathbf{0} \\
-\mathbf{V}_{2} & \mathbf{0} & (\overline{\mathbf{U}}-\mathbf{U})
\end{array}\right] \cdot\left[\begin{array}{c}
\delta \mathbf{u} \\
\delta \mathbf{v}_{1} \\
\delta \mathbf{v}_{2}
\end{array}\right]=\left[\begin{array}{c}
\mathbf{r}_{u} \\
\mathbf{r}_{v_{1}} \\
\mathbf{r}_{v_{2}}
\end{array}\right]
$$

Since the matrices on the second and third block-rows of (3.7) are diagonal, we can substitute $\delta \mathbf{v}_{1}=(\mathbf{U}-\underline{\mathbf{U}})^{-1}\left(\mathbf{r}_{v_{1}}-\mathbf{V}_{1} \delta \mathbf{u}\right)$ and $\delta \mathbf{v}_{2}=(\overline{\mathbf{U}}-\mathbf{U})^{-1}\left(\mathbf{r}_{v_{2}}+\mathbf{V}_{2} \delta \mathbf{u}\right)$ into the first block-row to obtain the reduced system

$$
\left[\mathbf{A}+(\mathbf{U}-\underline{\mathbf{U}})^{-1} \mathbf{V}_{1}+(\overline{\mathbf{U}}-\mathbf{U})^{-1} \mathbf{V}_{2}\right] \delta \mathbf{u}=\mathbf{r}
$$

with

$$
\mathbf{r}=\mathbf{r}_{u}+(\mathbf{U}-\underline{\mathbf{U}})^{-1} \mathbf{r}_{v_{1}}-(\overline{\mathbf{U}}-\mathbf{U})^{-1} \mathbf{r}_{2} .
$$

We note that the matrix of the reduced system (3.8) is symmetric positive definite, while the matrix of (3.7) is similar to the symmetric indefinite matrix

$$
\mathbf{C}=\left[\begin{array}{ccc}
\mathbf{A} & \mathbf{I} & \mathbf{I} \\
\mathbf{I} & -\mathbf{V}_{1}^{-1}(\mathbf{U}-\underline{\mathbf{U}}) & \mathbf{0} \\
\mathbf{I} & \mathbf{0} & -\mathbf{V}_{2}^{-1}(\overline{\mathbf{U}}-\mathbf{U})
\end{array}\right]
$$

If strict complementarity holds for at least one coordinate in the pair ( $\left.\widehat{\mathbf{u}}, \widehat{\mathbf{v}}_{1}\right)$ (resp. $\left.\left(\widehat{\mathbf{u}}, \widehat{\mathbf{v}}_{2}\right)\right)$, then the diagonal matrix $\mathbf{V}_{1}^{-1}(\mathbf{U}-\underline{\mathbf{U}})\left(\operatorname{resp} . \mathbf{V}_{2}^{-1}(\overline{\mathbf{U}}-\mathbf{U})\right.$ ) has increasingly 
small and/or large entries as the interior-point algorithm approaches the solution. It is easy to see that the largest eigenvalue of $\mathbf{C}$ is larger than any of the diagonal entries of $\mathbf{V}_{1}^{-1}(\mathbf{U}-\underline{\mathbf{U}})$ or $\mathbf{V}_{2}^{-1}(\overline{\mathbf{U}}-\mathbf{U})$, while the smallest (in absolute value) of its eigenvalues are $O(1)$. Therefore, the matrix $\mathbf{C}$, and hence the system (3.7) is ill-conditioned. Several equivalent reformulations of the system (3.7) are proposed in [4] (also see [17]). As in 2, 13, in this article we use the reduced form (3.8) which, for the problem under study, is symmetric positive definite. While (3.8) also suffers from the well known ill-conditioning of interior point methods, and in fact is even more ill-conditioned than (3.7) if $\beta \ll 1$, the condition number of (3.8) can be significantly reduced first by rescaling both the unknowns and the equations (left- and right-preconditioning), as shown below, and then by two-grid and multigrid preconditioning, as discussed in Sections 4 and 5 .

Given a vector $\mathbf{m}=\left[m_{1}, m_{2}, \ldots\right]^{T}$ we denote by $\mathbf{D}_{\mathbf{m}}$ the diagonal matrix with entries $D_{i i}=m_{i}$, and by $\mathbf{p} \cdot / \mathbf{m}$ the vector $\left[p_{1} / m_{1}, p_{2} / m_{2}, \ldots\right]^{T}$. With $\mathbf{A}$ as in (3.1), $\mathbf{w}=\mathbf{w}_{h}=\left[w_{1}, w_{2}, \ldots, w_{N_{h}}\right]^{T}$, and

$$
\mathbf{m}=\mathbf{v}_{1} \cdot /(\mathbf{u}-\underline{\mathbf{u}})+\mathbf{v}_{2} \cdot /(\overline{\mathbf{u}}-\mathbf{u}),
$$

the reduced system (3.8) can be written as

$$
\left(\mathbf{D}_{\mathbf{m}+\beta \mathbf{w}}+\mathbf{K}^{T} \mathbf{W K}\right) \delta \mathbf{u}=\mathbf{r} .
$$

Left-multiplication with $\mathbf{W}^{-1}$ further yields

$$
\left(\mathbf{D}_{(\mathbf{m} / \mathbf{w})+\beta \mathbf{e}}+\mathbf{W}^{-1} \mathbf{K}^{T} \mathbf{W K}\right) \delta \mathbf{u}=\mathbf{W}^{-1} \mathbf{r} .
$$

Let $\mathbf{p}=\sqrt{(\mathbf{m} / \mathbf{w})+\beta \mathbf{e}}$ (component-wise). By rescaling $\delta \mathbf{u}^{\prime}=\mathbf{D}_{\mathbf{p}} \delta \mathbf{u}$, and factoring out $\mathbf{D}_{\mathbf{p}}$ in (3.10), the system becomes

$$
\left(\mathbf{I}+\mathbf{W}^{-1} \mathbf{L}^{T} \mathbf{W L}\right) \delta \mathbf{u}^{\prime}=\mathbf{D}_{1 \cdot / \mathbf{p}} \mathbf{W}^{-1} \mathbf{r}
$$

where

$$
\mathbf{L}=\mathbf{L}_{h}=\mathbf{K}_{h} \mathbf{D}_{1 \cdot / \mathbf{p}},
$$

and we used the commutation of the diagonal matrices $\mathbf{W}^{-1}$ and $\mathbf{D}_{1 \cdot / \mathbf{p}}$. We prefer to write (3.11) in compact form as

$$
(\mathbf{I}+\mathbf{H}) \delta \mathbf{u}^{\prime}=\mathbf{r}^{\prime},
$$

with

$$
\mathbf{H}=\mathbf{H}_{h} \stackrel{\text { def }}{=}\left(\mathbf{W}_{h}\right)^{-1} \mathbf{L}_{h}^{T} \mathbf{W}_{h} \mathbf{L}_{h},
$$

and $\mathbf{r}^{\prime}=\mathbf{D}_{1 \cdot / \mathbf{p}} \mathbf{W}^{-1} \mathbf{r}$. Note that the matrix in (3.9) is symmetric positive definite, while the matrix $\mathbf{H}$ is symmetric (and positive definite) with respect to the $\mathbf{W}$-dot product. Furthermore, it is easy to see that $\|\mathbf{H}\|=O\left(\beta^{-1}\|\mathbf{K}\|^{2}\right)$ which implies that

$$
\operatorname{cond}(\mathbf{I}+\mathbf{H})=O\left(\beta^{-1}\|\mathbf{K}\|^{2}\right),
$$

independently of the mesh parameter $h$. However, for the model problems considered, the matrix $(\mathbf{I}+\mathbf{H})$ is dense (in standard representation), and therefore for large-scale problems it cannot be formed and/or stored. Matrix-vector multiplication can be 
performed at a cost equivalent to two applications of the matrix $\mathbf{K}$, hence residual computations are expensive. So (3.12) has to be solved using iterative methods, and for increased efficiency we need high-quality, matrix-free preconditioners. As it turns out, it is the system (3.12) rather than (3.9) that renders itself to good multigrid preconditioning. In the next sections we develop a multigrid preconditioner for (3.12) under the assumption that $\mathbf{m}$ represents a positive and relatively "smooth" function $\mu_{h}$.

4. The two-grid preconditioner. In this section we develop and analyze a two-grid preconditioner for the linear system (3.12). The work relies on the multigrid techniques developed by Drăgănescu and Dupont in [13. for (1.5). As will be shown, the constructed preconditioner has, under certain hypotheses, optimal order quality with respect to the discretization parameter $h$.

4.1. Algorithm design. For the purpose of algorithm design and analysis it is advantageous to regard (3.12) as an equation in $\mathcal{V}_{h}$ rather than $\mathbb{R}^{N_{h}}$, so we have to identify the operator in $\mathfrak{L}\left(\mathcal{V}_{h}\right)$ that is represented by $\mathbf{H}_{h}$. First we define for $\lambda \in L^{\infty}(\Omega)$ the multiplication-by- $\lambda$ operator $\mathcal{D}_{\lambda}: L^{2} \rightarrow L^{2}$ by

$$
\mathcal{D}_{\lambda} u=\lambda u,
$$

and its discrete version $\mathcal{D}_{\lambda}^{h} \in \mathfrak{L}\left(\mathcal{V}_{h}\right)$ by

$$
\mathcal{D}_{\lambda}^{h} u=\mathcal{I}_{h} \mathcal{D}_{\lambda} u .
$$

Given a vector $\mathbf{m} \in \mathbb{R}^{N_{h}}$ we define a function $\mu_{h} \in \mathcal{V}_{h}$ by setting $\mu_{h}\left(P_{i}^{h}\right)=\mathbf{m}_{i}$, $i=1, \ldots, N_{h}$; it follows that the diagonal matrix $\mathbf{D}_{(\mathbf{m} / \mathbf{w})+\beta}$ represents the operator

$$
\mathcal{D}_{\left(\mu_{h} / w_{h}\right)+\beta}^{h}=\mathcal{I}_{h} \mathcal{D}_{\left(\mu_{h} / w_{h}\right)+\beta} .
$$

To simplify notation let

$$
\lambda_{h}=\left(\mu_{h} / w_{h}\right)+\beta .
$$

Then $\mathbf{L}_{h}=\mathbf{K}_{h} \mathbf{D}_{1 \cdot / \mathbf{p}}$ represents the operator

$$
\mathcal{L}_{h}=\mathcal{K}_{h} \mathcal{D}_{1 / \sqrt{\lambda_{h}}}^{h} .
$$

If we denote by $\mathcal{L}_{h}^{*}$ the dual of $\mathcal{L}_{h}$ with respect to the $\langle\cdot, \cdot\rangle_{h}$-inner product, that is,

$$
\left\langle\mathcal{L}_{h} u, v\right\rangle_{h}=\left\langle u, \mathcal{L}_{h}^{*} v\right\rangle_{h}, \quad \forall u, v \in \mathcal{V}_{h},
$$

then $\mathcal{L}_{h}^{*}$ is represented by $\mathbf{W}_{h}^{-1} \mathbf{L}_{h}^{T} \mathbf{W}_{h}$, so $\mathbf{H}_{h}=\left(\mathbf{W}_{h}\right)^{-1} \mathbf{L}_{h}^{T} \mathbf{W}_{h} \mathbf{L}_{h}$ represents the operator

$$
\mathcal{H}_{h} \stackrel{\text { def }}{=} \mathcal{L}_{h}^{*} \mathcal{L}_{h} .
$$

Hence, the operator we need to invert for solving (3.12) is

$$
\mathcal{G}_{h}=I+\mathcal{H}_{h} .
$$

Note that the operator $\mathcal{G}_{h}$ is symmetric with respect to $\langle\cdot, \cdot\rangle_{h}$, i.e., $\mathcal{G}_{h}=\mathcal{G}_{h}^{*}$. 
The idea behind the proposed two-grid preconditioner for $\mathcal{G}_{h}$ lies in the "smoothing" properties of $\mathcal{L}_{h}$. More precisely, we regard $\mathcal{L}_{h}$ as a discretization of

$$
\mathcal{L}=\mathcal{K} \mathcal{D}_{1 / \sqrt{\lambda}}
$$

for some function $\lambda$ for which

$$
\mathcal{I}_{h}(\lambda)=\lambda_{h}
$$

If $\lambda$ (assumed to be $\geqslant \beta>0$ ) is relatively smooth (e.g., it can always be chosen to be $C^{2}$ ), then the multiplication operator $\mathcal{D}_{1 / \sqrt{\lambda}}$ is neither smoothing nor roughening, so the follow-on application of $\mathcal{K}$ results in smoothing. An alternative point of view is that $\mathcal{D}_{1 / \sqrt{\lambda}}$ is bounded in $\mathfrak{L}\left(L^{2}\right)$, and if $\mathcal{K}$ is compact, then $\mathcal{K} \mathcal{D}_{1 / \sqrt{\lambda}}$ is also compact. Hence, it is natural to assume that $\mathcal{H}_{h}=\mathcal{L}_{h}^{*} \mathcal{L}_{h}$ is "smoothing", even though $\mathcal{L}_{h}^{*}$ has no direct connection with the dual of $\mathcal{K D}_{1 / \sqrt{\lambda}}$ in $\mathfrak{L}\left(L^{2}\right)$.

We consider the $L^{2}$-orthogonal splitting of the discrete space

$$
\mathcal{V}_{h}=\mathcal{V}_{2 h} \oplus \mathcal{W}_{2 h}
$$

and let $\pi=\pi_{2 h}$ be the $L^{2}$-projector onto $\mathcal{V}_{2 h}$. Following [33, 23, 12, 13, we propose

$$
\mathcal{N}_{h}=\rho+\mathcal{G}_{2 h} \pi
$$

as a two-grid preconditioner, where $\rho=\rho_{2 h}=I-\pi_{2 h}$ is the projector on $\mathcal{W}_{2 h}$, and the coarse function $\lambda_{2 h}$ entering the definition of $\mathcal{G}_{2 h}$ is given by

$$
\lambda_{2 h}=\mathcal{I}_{h} \lambda_{h}
$$

The operator $\mathcal{N}_{h}$ can also be regarded as an additive Schwartz preconditioner with respect to the splitting (4.5) (see [23]). Moreover, the inverse of $\mathcal{N}_{h}$ is given by

$$
\mathcal{S}_{h} \stackrel{\text { def }}{=} \mathcal{N}_{h}^{-1}=\rho+\mathcal{G}_{2 h}^{-1} \pi
$$

For developing a multigrid algorithm of comparable quality with the two-grid preconditioner we follow the same strategy as in [12, 13], which we briefly outline in Section 5 .

As shown in the analysis, the use of the $L^{2}$-projector $\pi_{2 h}$ in the definition of $\mathcal{S}_{h}$, as opposed to other projectors or restriction operators turns out to be critical for the quality of the preconditioner. Unfortunately $\pi_{2 h}$ is not symmetric with respect to $\langle\cdot, \cdot\rangle_{h}$, therefore $\mathcal{N}_{h}$ and $\mathcal{S}_{h}$ are symmetric neither with respect to $\langle\cdot, \cdot\rangle_{h}$ nor to $\langle\cdot, \cdot\rangle_{L^{2}}$, but they are almost symmetric. At the root of this problem lies the use of meshdependent norms in the formulation of the discrete optimization problem (2.5), which in turn was necessary for the linear systems inner to the interior point algorithms to be of the form (3.9), that is, to have a diagonal matrix $\mathbf{D}_{\mathbf{m}+\beta \mathbf{w}}$ added to $\mathbf{K}^{T} \mathbf{W K}$. Had we used the exact $L^{2}$-norm in the discrete formulation (2.5), then the matrix in the system (3.9) would have had the form $\mathbf{D}_{\mathbf{m}}+\beta \mathbf{M}+\mathbf{K}^{T} \mathbf{M K}$, where $\mathbf{M}$ is the mass matrix, and this form is less convenient for preconditioning.

In order to describe the matrices representing $\mathcal{N}_{h}$ and $\mathcal{S}_{h}$ we consider the prolongation operator $\mathbf{J}_{h} \in M_{N_{h} \times N_{2 h}}$ representing the natural embedding of $\mathcal{V}_{2 h}$ into $\mathcal{V}_{h}$, and we define the restriction $\mathbf{R}_{2 h} \in M_{N_{2 h} \times N_{h}}$ by $\mathbf{R}_{2 h}=2^{-d} \mathbf{J}_{h}^{T}, d$ being the dimension of the ambient space (here $d=2$ ). Then $\pi_{2 h}$ is represented by the matrix

$$
\mathbf{\Pi}_{2 h}=\mathbf{M}_{2 h}^{-1} \cdot \mathbf{R}_{2 h} \cdot \mathbf{M}_{h}
$$


where $\mathbf{M}_{h}$ (resp. $\mathbf{M}_{2 h}$ ) is the rescaled mass matrix on the fine (resp. coarse) mesh, defined by $\left(\mathbf{M}_{h}\right)_{i j}=h^{-d}\left\langle\varphi_{i}^{h}, \varphi_{j}^{h}\right\rangle$. Note that $\boldsymbol{\Pi}_{2 h}$ is a matrix of type $N_{2 h} \times N_{h}$, and that $\mathbf{M}_{2 h}=\mathbf{R}_{2 h} \cdot \mathbf{M}_{h} \cdot \mathbf{J}_{h}$. Furthermore, the square $N_{h} \times N_{h}$ projection matrix is given by $\mathbf{P}_{h}=\mathbf{J}_{h} \cdot \boldsymbol{\Pi}_{2 h}$, so that $\mathbf{P}_{h}^{2}=\mathbf{P}_{h}$. The projector $\rho=(I-\pi)$ is represented by the matrix $\mathbf{Q}_{h}=\left(\mathbf{I}-\mathbf{P}_{h}\right) \in M_{N_{h} \times N_{h}}$. Finally, $\mathcal{S}_{h}$ is represented by

$$
\mathbf{S}_{h}=\mathbf{Q}_{h}+\mathbf{J}_{h} \mathbf{G}_{2 h}^{-1} \mathbf{\Pi}_{2 h} .
$$

Oftentimes in practice the exact projection $\boldsymbol{\Pi}_{2 h}$ in (4.9) is replaced by the restriction $\mathbf{R}_{2 h}$, and $\mathbf{Q}_{h}$ is taken to be $\left(\mathbf{I}-\mathbf{R}_{2 h}\right)$. While for certain problems this is a viable option [2, for the applications considered in this work the quality of the preconditioner is significanly diminished by this change.

4.2. Algorithm analysis. Our analysis consists of evaluating the quality of the two-grid preconditioner by eventually estimating the spectral distance $d_{\sigma}\left(\mathcal{S}_{h}, \mathcal{G}_{h}^{-1}\right)$ (Theorem 4.9), and is performed over three steps. First we evaluate the norm-distance $\left\|\mathcal{G}_{h}-\mathcal{N}_{h}\right\|$ under the assumption that $\mathcal{H}_{h}$ satisfies Condition 4.1 below. Second, we show that if $\mathcal{L}$ and $\mathcal{L}_{h}$ verify Condition 2.1, then $\mathcal{H}_{h}$ satisfies Condition 4.1, Third, we show that $\mathcal{L}, \mathcal{L}_{h}$ satisfy Condition 2.1 (SAC) with a constant $C(\mathcal{L})$ depending on $\lambda$ and on $C(\mathcal{K})$, that is, the constant associated to $\mathcal{K}, \mathcal{K}_{h}$ satisfying SAC. For specific applications, the fact that $\mathcal{K}, \mathcal{K}_{h}$ satifsy $\mathrm{SAC}$ is normally verifiable, as is shown in Section 6.

Condition 4.1. The operators $\mathcal{H}_{h}$ are symmetric with respect to $\langle\cdot, \cdot\rangle_{h}$, positive semidefinite, and uniformly bounded with respect to $h \in I$, that is, there exists a constant $C(\mathcal{H})>0$ independent on $h$ such that

$$
\left\|\mathcal{H}_{h}\right\|_{h} \leqslant C(\mathcal{H}), \quad \forall h \in I .
$$

Moreover, there exists $p>0$ so that

$$
\left\|\left(\mathcal{H}_{h}-\mathcal{H}_{2 h} \pi_{2 h}\right) u\right\| \leqslant C(\mathcal{H}) h^{p}\|u\|, \text { for all } u \in \mathcal{V}_{h}, h \in I .
$$

For linear splines the optimal approximation order is $p=2$, but for certain problems and discretizations the actual rate may be suboptimal.

Lemma 4.2. If $\mathcal{H}_{h}$ satisfies Condition 4.1, then

$$
\left\|\mathcal{G}_{h}-\mathcal{N}_{h}\right\| \leqslant C(\mathcal{H}) h^{p} .
$$

Proof. This result is an immediate consequence of (4.11), since

$$
\mathcal{G}_{h}-\mathcal{N}_{h}=\left(I+\mathcal{H}_{h}\right)-\left(\rho+\left(I+\mathcal{H}_{2 h}\right) \pi\right)=\mathcal{H}_{h}-\mathcal{H}_{2 h} \pi .
$$

Verifying that (4.11) of Condition 4.1 holds under some general hypotheses is nontrivial for this problem due to the presence of multiple inner products that have to be taken into consideration. More precisely, if $\mathcal{L}_{h}^{*}$ were the dual of $\mathcal{L}_{h}$ with respect to $\langle\cdot, \cdot\rangle$ instead of $\langle\cdot, \cdot\rangle_{h}$, then Condition 4.1 would follow from the approximability of $\mathcal{L}$ by $\mathcal{L}_{h}$ together with the smoothing properties of $\mathcal{L}$, as is shown in 13] (proof of Theorem 4.1). Hence a natural requirement is that $\langle\cdot, \cdot\rangle_{h}$ approximates $\langle\cdot, \cdot\rangle$ well.

Lemma 4.3. With $w_{h}$ chosen as in (2.1) there exists a constant $C=C\left(\mathcal{T}_{0}\right)>0$ independent of $h$ such that

$$
\left|\langle u, v\rangle_{h}-\langle u, v\rangle\right| \leqslant C h^{2}\|u\|_{H^{1}(\Omega)} \cdot\|v\|_{H^{1}(\Omega)}, \forall u, v \in \mathcal{V}_{h} .
$$


Proof. By Theorem 4.4.4 in [9], given $d=2$, there exists a constant $C>0$ that depends on the "chunkiness" of the initial triangulation $\mathcal{T}_{0}$ but is independent of $h$ and of $T \in \mathcal{T}_{h}$, so that for all $u, v \in \mathcal{V}_{h}$

$$
\left\|u v-\mathcal{I}_{h}(u v)\right\|_{L^{1}(T)} \leqslant C h^{2}|u v|_{W_{1}^{2}(T)} \stackrel{u, v}{=}{ }^{\text {linear on } T} C h^{2} \int_{T}|\nabla u \cdot \nabla v| .
$$

After summing over all triangles $T \in \mathcal{T}_{h}$ we obtain

$$
\left\|u v-\mathcal{I}_{h}(u v)\right\|_{L^{1}(\Omega)} \leqslant C h^{2}\|\nabla u \cdot \nabla v\|_{L^{1}(\Omega)} \leqslant C h^{2}|u|_{H^{1}(\Omega)} \cdot|v|_{H^{1}(\Omega)} .
$$

The conclusion follows from

$$
\left|\langle u, v\rangle-\langle u, v\rangle_{h}\right|=\left|\int_{\Omega}\left(u v-\mathcal{I}_{h}(u v)\right)\right| \leqslant\left\|u v-\mathcal{I}_{h}(u v)\right\|_{L^{1}(\Omega)} .
$$

Throughout this section we denote by $C(\mathcal{L})$ a generic constant that is proportional to the constant $C(\mathcal{L})$ of Condition 2.1 if the proportionality depends only on the domain $\Omega$ and the initial triangulation $\mathcal{T}_{0}$. We define the restriction operator $\mathcal{R}_{2 h}^{w}: \mathcal{V}_{h} \rightarrow \mathcal{V}_{2 h}$ by

$$
\left\langle\iota_{h} u, v\right\rangle_{h}=\left\langle u, \mathcal{R}_{2 h}^{w} v\right\rangle_{2 h}, \forall u \in \mathcal{V}_{2 h}, v \in \mathcal{V}_{h},
$$

where $\iota_{h}: \mathcal{V}_{2 h} \rightarrow \mathcal{V}_{h}$ is the inclusion operator. It follows that $\mathcal{R}_{2 h}^{w}$ is uniformly bounded with respect to $h \in I$, that is, there exists $C$ independent of $h \in I$ so that

$$
\left\|\mathcal{R}_{2 h}^{w} u\right\| \leqslant C\|u\|, \quad \forall u \in \mathcal{V}_{h} .
$$

We call a triangulation $\mathcal{T}$ locally symmetric if for every vertex $P$ the union of triangles in $\mathcal{T}$ having $P$ as a corner is invariant with respect to the reflection through $P$ given by $r_{P}(x)=(2 P-x)$. If $\mathcal{T}$ is locally symmetric and $\varphi_{P}$ is the nodal basis function at $P$, then $\varphi_{P} \circ r_{P}=\varphi_{P}$. Furthermore, a simple calculation shows that for any linear map $L(x)=a_{1} x_{1}+a_{2} x_{2}$ we have

$$
\int_{\Omega} \varphi_{P}(x) L(x-P) d x=0 .
$$

Naturally, a uniform mesh is locally symmetric.

The following grouped results are either simple consequences of Condition 2.1 or extracted from [13].

Lemma 4.4. If $\mathcal{L}, \mathcal{L}_{h}$ satisfy Condition 2.1 there exist constants $C(\mathcal{L})$ and $C^{\prime}=C^{\prime}(\Omega)$ independent of $h$ such that the following hold:

(a) $H^{1}, L^{2}$ - uniform stability of $\mathcal{L}_{h}$ :

$$
\left\|\mathcal{L}_{h} u\right\|_{H^{m}(\Omega)} \leqslant C(\mathcal{L})\|u\|, \forall u \in \mathcal{V}_{h}, m=0,1 ;
$$

(b) smoothing of negative-index norm:

$$
\|\mathcal{L} u\| \leqslant C(\mathcal{L})\|u\|_{\widetilde{H}^{-m}}, \forall u \in \mathcal{V}_{h}, m=1,2 ;
$$

(c) negative-index norm approximation of the identity by $\pi_{2 h}, \mathcal{R}_{2 h}^{w}$ :

$$
\begin{gathered}
\left\|\left(I-\pi_{2 h}\right) u\right\|_{\widetilde{H}^{-2}(\Omega)} \leqslant C^{\prime} h^{2}\|u\|, \quad \forall u \in \mathcal{V}_{h} \\
\left\|\left(I-\mathcal{R}_{2 h}^{w}\right) u\right\|_{\widetilde{H}^{-p}(\Omega)} \leqslant C^{\prime} h^{p}\|u\|, \quad \forall u \in \mathcal{V}_{h},
\end{gathered}
$$


where $p=1$ on an unstructured grid, and $p=2$ on a locally symmetric grid;

(d) $\mathcal{L}$ diminishes high-frequencies:

$$
\begin{aligned}
\left\|\mathcal{L}\left(I-\pi_{2 h}\right) u\right\| \leqslant C(\mathcal{L}) h^{2}\|u\|, & \forall u \in \mathcal{V}_{h} ; \\
\left\|\mathcal{L}\left(I-\mathcal{R}_{2 h}^{w}\right) u\right\| \leqslant C(\mathcal{L}) h^{p}\|u\|, & \forall u \in \mathcal{V}_{h},
\end{aligned}
$$

where $p=1$ on an unstructured grid, and $p=2$ on a locally symmetric grid;

(e)

$$
\left|\langle\mathcal{L} u, \mathcal{L} v\rangle-\left\langle\mathcal{L}_{h} u, \mathcal{L}_{h} v\right\rangle\right| \leqslant C(\mathcal{L}) h^{2}\|u\| \cdot\|v\|, \forall u \in \mathcal{V}_{h}
$$

Proof. The stability conditions at (a) are direct consequences of (2.3) and (2.4), and (b) follows from (2.3) (see also [13. Corollary 6.2). The estimate (4.18) is a straightforward consequence of the Bramble-Hilbert Lemma [9], while (4.19) follows from Theorem 6.6 in 13 . (see Example 6.7 for the uniform mesh case). The inequalities at (d) follow from (b) and (c), and (e) follows from (2.4) and the uniform boundedness of $\mathcal{L}_{h}$. $\square$

Proposition 4.5. If the operators $\mathcal{L}, \mathcal{L}_{h}$ satisfy Condition 2.1 with the weights given by (2.1), then Condition 4.1 holds with $C(\mathcal{H})=C(\mathcal{L})$ and $p=2$ if the meshes are locally symmetric, or $p=1$ otherwise.

Proof. To simplify notation we write $\pi=\pi_{2 h}, \mathcal{R}=\mathcal{R}_{2 h}^{w}$. First we have

$$
\begin{aligned}
& \left\langle\mathcal{L}_{2 h}^{*} \mathcal{L}_{2 h} \pi u, v\right\rangle_{h}=\left\langle\mathcal{L}_{2 h}^{*} \mathcal{L}_{2 h} \pi u, \mathcal{R} v\right\rangle_{2 h}=\left\langle\mathcal{L}_{2 h} \pi u, \mathcal{L}_{2 h} \mathcal{R} v\right\rangle_{2 h}, \text { and } \\
& \left\langle\mathcal{L}_{h}^{*} \mathcal{L}_{h} u, v\right\rangle_{h}=\left\langle\mathcal{L}_{h} u, \mathcal{L}_{h} v\right\rangle_{h} .
\end{aligned}
$$

Therefore

$$
\begin{aligned}
& \left|\left\langle\mathcal{L}_{2 h}^{*} \mathcal{L}_{2 h} \pi u, v\right\rangle_{h}-\left\langle\mathcal{L}_{h}^{*} \mathcal{L}_{h} u, v\right\rangle_{h}\right|=\left|\left\langle\mathcal{L}_{2 h} \pi u, \mathcal{L}_{2 h} \mathcal{R} v\right\rangle_{2 h}-\left\langle\mathcal{L}_{h} u, \mathcal{L}_{h} v\right\rangle_{h}\right| \\
& \leqslant \underbrace{\left|\left\langle\mathcal{L}_{2 h} \pi u, \mathcal{L}_{2 h} \mathcal{R} v\right\rangle_{2 h}-\left\langle\mathcal{L}_{2 h} \pi u, \mathcal{L}_{2 h} \mathcal{R} v\right\rangle\right|}_{A_{1}}+\underbrace{\left|\left\langle\mathcal{L}_{2 h} \pi u, \mathcal{L}_{2 h} \mathcal{R} v\right\rangle-\langle\mathcal{L} \pi u, \mathcal{L} \mathcal{R} v\rangle\right|}_{A_{2}} \\
& +\underbrace{|\langle\mathcal{L} \pi u, \mathcal{L} \mathcal{R} v\rangle-\langle\mathcal{L} u, \mathcal{L} v\rangle|}_{A_{4}} \\
& +\underbrace{\left|\langle\mathcal{L} u, \mathcal{L} v\rangle-\left\langle\mathcal{L}_{h} u, \mathcal{L}_{h} v\right\rangle\right|}_{A_{5}}+\underbrace{\left|\left\langle\mathcal{L}_{h} u, \mathcal{L}_{h} v\right\rangle-\left\langle\mathcal{L}_{h} u, \mathcal{L}_{h} v\right\rangle_{h}\right|} .
\end{aligned}
$$

For $A_{1}$ we have

$$
\begin{aligned}
& A_{1} \stackrel{\stackrel{4.13}{\leqslant}}{\leqslant} C(\mathcal{L}) h^{2}\left\|\mathcal{L}_{2 h} \pi u\right\|_{H^{1}(\Omega)} \cdot\left\|\mathcal{L}_{2 h} \mathcal{R} v\right\|_{H^{1}(\Omega)} \stackrel{4.16)}{\leqslant} C(\mathcal{L}) h^{2}\|\pi u\| \cdot\|\mathcal{R} v\| \\
& \quad \stackrel{4.14}{\leqslant} C(\mathcal{L}) h^{2}\|u\| \cdot\|v\|,
\end{aligned}
$$

and a similar estimate holds for $A_{5}$. Also (4.22) implies that

$$
\max \left(A_{2}, A_{4}\right) \leqslant C(\mathcal{L}) h^{2}\|u\| \cdot\|v\|
$$

For $A_{3}$ we have

$$
A_{3} \leqslant|\langle\mathcal{L}(\pi-I) u, \mathcal{L} \mathcal{R} v\rangle|+|\langle\mathcal{L} u,(\mathcal{L}(\mathcal{R}-I) v)\rangle| \stackrel{4.201}{\leqslant} C(\mathcal{L}) h^{p}\|u\| \cdot\|v\|
$$


Since $p \leqslant 2, A_{3}$ is the weak link, and we have

$$
\left|\left\langle\left(\mathcal{H}_{2 h} \pi-\mathcal{H}_{h}\right) u, v\right\rangle_{h}\right|=\left|\left\langle\left(\mathcal{L}_{2 h}^{*} \mathcal{L}_{2 h} \pi-\mathcal{L}_{h}^{*} \mathcal{L}_{h}\right) u, v\right\rangle_{h}\right| \leqslant C(\mathcal{L}) h^{p}\|u\| \cdot\|v\|, \quad \forall v \in \mathcal{V}_{h},
$$

and the conclusion follows from the equivalence of $\|\cdot\|$ with $\|\cdot\|_{h}$. $\square$

For $\rho \in W_{\infty}^{2}(\Omega)$ we denote by

$$
\|\rho\|_{W_{\infty}^{2}(\Omega) / \mathbb{R}}=\max _{1 \leqslant|\alpha| \leqslant 2}\left\|\partial^{\alpha} \rho\right\|_{L^{\infty}(\Omega)},
$$

which effectively is the norm on the quotient space $W_{\infty}^{2}(\Omega) / \mathbb{R}$.

Lemma 4.6. If $\rho \in W_{\infty}^{2}(\Omega)$ then there exists a constant $C>0$ independent of $\rho, h$ so that

$$
\left\|\left(\mathcal{D}_{\rho}-\mathcal{D}_{\rho_{h}}^{h}\right) u\right\| \leqslant C h^{2}\|\rho\|_{W_{\infty}^{2}(\Omega) / \mathbb{R}}\|u\|_{H^{1}(\Omega)}, \quad \forall u \in \mathcal{V}_{h}, h \in I,
$$

where $\rho_{h}=\mathcal{I}_{h}(\rho)$.

Proof. Note first that $\mathcal{D}_{\rho}^{h}=\mathcal{D}_{\rho_{h}}^{h}=\mathcal{I}_{h} \mathcal{D}_{\rho}$, since only the node values of $\rho$ enter the definition of the operator $\mathcal{D}_{\rho}^{h}$. Given $u \in \mathcal{V}_{h}$, for each triangle $T \in \mathcal{T}_{h}$ we have

$$
|\rho u|_{H^{2}(T)} \leqslant C\|\rho\|_{W_{\infty}^{2}(T) / \mathbb{R}} \cdot\|u\|_{H^{1}(T)},
$$

because $u$ is linear on $T$. Therefore

$$
\begin{aligned}
\left\|\left(\mathcal{D}_{\rho}-\mathcal{D}_{\rho}^{h}\right) u\right\| & =\left\|\rho u-\mathcal{I}_{h}(\rho u)\right\| \leqslant C h^{2}\left(\sum_{T \in \mathcal{T}_{h}}|\rho u|_{H^{2}(T)}^{2}\right)^{\frac{1}{2}} \\
& \leqslant C h^{2}\left(\sum_{T \in \mathcal{T}_{h}}\|\rho\|_{W_{\infty}^{2}(T) / \mathbb{R}}^{2}\|u\|_{H^{1}(T)}^{2}\right)^{\frac{1}{2}} \leqslant C h^{2}\|\rho\|_{W_{\infty}^{2}(\Omega) / \mathbb{R}}\|u\|_{H^{1}(\Omega)} .
\end{aligned}
$$

LEMma 4.7. If $\rho \in W_{\infty}^{2}(\Omega)$ then there exists a constant $C>0$ independent of $\rho, h$ so that

$$
\left\|\left(\mathcal{D}_{\rho}-\mathcal{D}_{\rho_{h}}^{h}\right) u\right\|_{\widetilde{H}^{-1}(\Omega)} \leqslant C h^{p}\|\rho\|_{W_{\infty}^{p}(\Omega) / \mathbb{R}}\|u\|, \quad \forall u \in \mathcal{V}_{h}, h \in I,
$$

where $\rho_{h}=\mathcal{I}_{h}(\rho)$, and $p=2$ if the mesh is locally symmetric, otherwise $p=1$.

Proof. We focus on the situation when the mesh is locally symmetric and leave the general case as an exercise. Let $u \in \mathcal{V}_{h}, v \in H_{0}^{1}(\Omega)$ be arbitrary, and denote by $S_{i}=\operatorname{supp}\left(\varphi_{i}^{h}\right)$. The constant $C$ is assumed to be independent of $u, v, \rho, h$. First remark that each triangle in $\mathcal{T}_{h}$ lies in at most three of the sets $S_{i}$ and that

$$
\operatorname{diam}\left(S_{i}\right) \leqslant C h, \quad 1 \leqslant i \leqslant N_{h}
$$

due to the quasi-uniformity for the meshes. Also note that

$$
\rho \varphi_{i}^{h}-\mathcal{I}_{h}\left(\rho \varphi_{i}^{h}\right)=\varphi_{i}^{h}\left(\rho-\rho\left(P_{i}^{h}\right)\right), \quad 1 \leqslant i \leqslant N_{h} .
$$

Further we define $v_{i}=\frac{1}{\operatorname{area}\left(S_{i}\right)} \int_{S_{i}} v$, for $1 \leqslant i \leqslant N_{h}$, and $u_{i}=u\left(P_{i}^{h}\right)$. Then $u=\sum_{i=1}^{N_{h}} u_{i} \varphi_{i}^{h}$ and

$$
\begin{array}{r}
\left|\left\langle\left(\mathcal{D}_{\rho}-\mathcal{D}_{\rho_{h}}^{h}\right) u, v\right\rangle\right|=\left|\int_{\Omega}\left(\rho u-\mathcal{I}_{h}(\rho u)\right) v\right| \stackrel{\sqrt[4.27]{=}}{=}\left|\sum_{i=1}^{N_{h}} u_{i}\left(\int_{S_{i}} \varphi_{i}^{h}\left(\rho-\rho\left(P_{i}^{h}\right)\right) v\right)\right| \\
\leqslant \sum_{i=1}^{N_{h}}\left|u_{i}\right|\left(\left|\int_{S_{i}} \varphi_{i}^{h}\left(\rho-\rho\left(P_{i}^{h}\right)\right)\left(v-v_{i}\right)\right|+\mid \int_{S_{i}} \varphi_{i}^{h}\left(\rho-\rho\left(P_{i}^{h}\right)\right) v_{i}\right) \mid .
\end{array}
$$


For the first term in the sum above

$$
\begin{aligned}
\left|\int_{S_{i}} \varphi_{i}^{h}\left(\rho-\rho\left(P_{i}^{h}\right)\right)\left(v-v_{i}\right)\right| & \leqslant\left\|\varphi_{i}^{h}\right\| \cdot\left\|\rho-\rho\left(P_{i}^{h}\right)\right\|_{L^{\infty}\left(S_{i}\right)} \cdot\left\|v-v_{i}\right\|_{L^{2}\left(S_{i}\right)} \\
& \stackrel{(4.26)}{\leqslant} C h^{2}\left\|\varphi_{i}^{h}\right\| \cdot|\rho|_{W_{\infty}^{1}\left(S_{i}\right)} \cdot|v|_{H^{1}\left(S_{i}\right)} .
\end{aligned}
$$

For the second term in the sum we take advantage of the local grid symmetry:

$$
\begin{aligned}
\left|\int_{S_{i}} \varphi_{i}^{h}\left(\rho-\rho\left(P_{i}^{h}\right)\right) v_{i}\right| & \stackrel{\sqrt[4.15]{=}}{=}\left|\int_{S_{i}} v_{i} \varphi_{i}^{h}(x)\left(\rho(x)-\rho\left(P_{i}^{h}\right)-d \rho_{P_{i}^{h}}\left(x-P_{i}^{h}\right)\right) d x\right| \\
& \leqslant\left\|v_{i} \varphi_{i}^{h}\right\|_{L^{1}\left(S_{i}\right)} \cdot\left\|\rho-\rho\left(P_{i}^{h}\right)-d \rho_{P_{i}^{h}}\left(x-P_{i}^{h}\right)\right\|_{L^{\infty}\left(S_{i}\right)} \\
& \leqslant C h^{2}\left\|v_{i}\right\|_{L^{2}\left(S_{i}\right)}\left\|\varphi_{i}^{h}\right\| \cdot|\rho|_{W_{\infty}^{2}\left(S_{i}\right)} .
\end{aligned}
$$

Since $\left\|v_{i}\right\|_{L^{2}\left(S_{i}\right)} \leqslant\|v\|_{L^{2}\left(S_{i}\right)}$ we now have

$$
\begin{aligned}
& \left|\int_{\Omega}\left(\rho u-\mathcal{I}_{h}(\rho u)\right) v\right| \\
& \leqslant C h^{2} \sum_{i=1}^{N_{h}}\left|u_{i}\right|\left\|\varphi_{i}^{h}\right\|\left(|\rho|_{W_{\infty}^{1}\left(S_{i}\right)} \cdot|v|_{H^{1}\left(S_{i}\right)}+\|v\|_{L^{2}\left(S_{i}\right)}|\rho|_{W_{\infty}^{2}\left(S_{i}\right)}\right) \\
& \leqslant C h^{2}\|\rho\|_{W_{\infty}^{2}(\Omega) / \mathbb{R}} \sum_{i=1}^{N_{h}}\left|u_{i}\right| \cdot\left\|\varphi_{i}^{h}\right\| \cdot\|v\|_{H^{1}\left(S_{i}\right)} \\
& \leqslant C h^{2}\|\rho\|_{W_{\infty}^{2}(\Omega) / \mathbb{R}}\left(\sum_{i=1}^{N_{h}}\left|u_{i}\right|^{2} \cdot\left\|\varphi_{i}^{h}\right\|^{2}\right)^{\frac{1}{2}} \cdot\left(\sum_{i=1}^{N_{h}}\|v\|_{H^{1}\left(S_{i}\right)}^{2}\right)^{\frac{1}{2}} \\
& \leqslant C h^{2}\|\rho\|_{W_{\infty}^{2}(\Omega) / \mathbb{R}}\|u\| \cdot\|v\|_{H^{1}(\Omega)},
\end{aligned}
$$

where for the last inequality we used the quasi-uniformity of the mesh and the fact the each triagle is in at most three of the sets $S_{i}$. The conclusion follows after dividing by $\|v\|_{H^{1}(\Omega)}$ and taking the sup over all $v \in H_{0}^{1}(\Omega)$.

Proposition 4.8. If the operators $\mathcal{K}, \mathcal{K}_{h}$ satisfy Condition 2.1 with $p=2$ on the locally-symmetric meshes $\mathcal{T}_{h}$ with the weights given by (2.1), then $\mathcal{L}, \mathcal{L}_{h}$ also satisfy Condition 2.1 with

$$
C(\mathcal{L})=C(\mathcal{K})\left\|\lambda^{-\frac{1}{2}}\right\|_{W_{\infty}^{2}(\Omega)}
$$

If the meshes are not locally-symmetric, then the power of $h$ in Condition $2.1[\mathrm{~b}]$ for the operators $\mathcal{L}, \mathcal{L}_{h}$ is 1 for both $m=0,1$.

Proof. Note that for $\rho \in L^{\infty}(\Omega),\left\|\mathcal{D}_{\rho} u\right\| \leqslant\|\rho\|_{L^{\infty}(\Omega)} \cdot\|u\|$, therefore

$$
\left\|\mathcal{K} \mathcal{D}_{1 / \sqrt{\lambda}} u\right\|_{H^{m}(\Omega)} \leqslant C(\mathcal{K})\left\|\mathcal{D}_{1 / \sqrt{\lambda}} u\right\| \leqslant C(\mathcal{K})\left\|\lambda^{-\frac{1}{2}}\right\|_{L^{\infty}(\Omega)} \cdot\|u\|, \quad m=0,1,2,
$$

which implies the smoothing condition (2.3). For $u \in \mathcal{V}_{h}$

$$
\begin{aligned}
& \left\|\left(\mathcal{K} \mathcal{D}_{1 / \sqrt{\lambda}}-\mathcal{K}_{h} \mathcal{D}_{1 / \sqrt{\lambda}}^{h}\right) u\right\|_{H^{1}(\Omega)} \\
& \quad \leqslant \mathcal{K}\left(\mathcal{D}_{1 / \sqrt{\lambda}}-\mathcal{D}_{1 / \sqrt{\lambda}}^{h}\right) u\left\|_{H^{1}(\Omega)}+\right\|\left(\mathcal{K}-\mathcal{K}_{h}\right) \mathcal{D}_{1 / \sqrt{\lambda}}^{h} u \|_{H^{1}(\Omega)} \\
& \stackrel{[2.31, \text { (2.4) }}{\leqslant} C(\mathcal{K})\left\|\left(\mathcal{D}_{1 / \sqrt{\lambda}}-\mathcal{D}_{1 / \sqrt{\lambda}}^{h}\right) u\right\|+C(\mathcal{K}) h\left\|\mathcal{D}_{1 / \sqrt{\lambda}}^{h} u\right\| \\
& \stackrel{[4.23)}{\leqslant} C(\mathcal{K}) h^{2}\left\|\lambda^{-\frac{1}{2}}\right\|_{W_{\infty}^{2}(\Omega) / \mathbb{R}}\|u\|_{H^{1}(\Omega)}+C(\mathcal{K}) h\left\|\lambda^{-\frac{1}{2}}\right\|_{L^{\infty}(\Omega)}\|u\| \\
& \quad C \quad C(\mathcal{K}) h\left\|\lambda^{-\frac{1}{2}}\right\|_{W_{\infty}^{2}(\Omega)}\|u\|,
\end{aligned}
$$


where in the last inequality we have used an inverse estimate. This proves (2.4) for $\mathcal{K}, \mathcal{K}_{h}$ with $m=1$ and concludes the proof for the non-locally-symmetric case. For $m=2$ and locally-symmetric mesh

$$
\begin{aligned}
& \left\|\left(\mathcal{K D}_{1 / \sqrt{\lambda}}-\mathcal{K}_{h} \mathcal{D}_{1 / \sqrt{\lambda}}^{h}\right) u\right\| \\
& \leqslant\left\|\mathcal{K}\left(\mathcal{D}_{1 / \sqrt{\lambda}}-\mathcal{D}_{1 / \sqrt{\lambda}}^{h}\right) u\right\|+\left\|\left(\mathcal{K}-\mathcal{K}_{h}\right) \mathcal{D}_{1 / \sqrt{\lambda}}^{h} u\right\| \\
& \stackrel{4.17 \text {, 2.4. }}{\leqslant} C(\mathcal{K})\left\|\left(\mathcal{D}_{1 / \sqrt{\lambda}}-\mathcal{D}_{1 / \sqrt{\lambda}}^{h}\right) u\right\|_{\widetilde{H}^{-1}(\Omega)}+C(\mathcal{K}) h^{2}\left\|\mathcal{D}_{1 / \sqrt{\lambda}}^{h} u\right\| \\
& \stackrel{4.25)}{\leqslant} C(\mathcal{K}) h^{2}\left\|\lambda^{-\frac{1}{2}}\right\|_{W_{\infty}^{2}(\Omega) / \mathbb{R}}\|u\|+C(\mathcal{K}) h^{2}\left\|\lambda^{-\frac{1}{2}}\right\|_{L^{\infty}(\Omega)}\|u\| \\
& \leqslant \quad C(\mathcal{K}) h^{2}\left\|\lambda^{-\frac{1}{2}}\right\|_{W_{\infty}^{2}(\Omega)}\|u\| .
\end{aligned}
$$

We conclude the analysis of the two-grid preconditioner with the computation of the spectral distance (SD) $d_{\sigma}$ between $\left(\mathcal{G}_{h}\right)^{-1}$ and the two-grid preconditioner $\mathcal{S}_{h}$ defined in (4.8). This step facilitates a smooth transition from the two-grid analysis to the multigrid analysis of the next section. Given a Hilbert space $(\mathcal{X},\langle\cdot, \cdot\rangle)$ we denote by $\mathfrak{L}_{+}(\mathcal{X})$ the set of operators with positive definite symmetric part:

$$
\mathfrak{L}_{+}(\mathcal{X})=\{T \in \mathfrak{L}(\mathcal{X}):\langle T u, u\rangle>0, \quad \forall u \in \mathcal{X} \backslash\{0\}\} .
$$

First we define the joined numerical range of $S, T \in \mathfrak{L}_{+}(\mathcal{X})$ by

$$
W(S, T)=\left\{\frac{\left\langle S_{\mathbb{C}} w, w\right\rangle}{\left\langle T_{\mathbb{C}} w, w\right\rangle}: w \in \mathcal{X}^{\mathbb{C}} \backslash\{0\}\right\},
$$

where $T_{\mathbb{C}}(u+\mathbf{i} v)=T(u)+\mathbf{i} T(v)$ is the complexification of $T$. Note that if $T$ is symmetric positive definite, then $W(S, T)$ is simply the numerical range of $T^{-\frac{1}{2}} S T^{-\frac{1}{2}}$. The spectral distance between $S, T \in \mathfrak{L}_{+}(\mathcal{X})$, introduced in [13] as a measure of spectral equivalence between $S$ and $T$, is defined by

$$
d_{\sigma}(S, T)=\sup \{|\ln z|: z \in W(S, T)\},
$$

where $\ln$ is the branch of the logarithm corresponding to $\mathbb{C} \backslash(-\infty, 0]$. Following Lemma 3.2 in [13, if $W(S, T) \subseteq \mathcal{B}_{\alpha}(1)=\{z \in \mathbb{C}:|z-1|<\alpha\}$ with $\alpha \in(0,1)$, then

$$
d_{\sigma}(S, T) \leqslant \frac{|\ln (1-\alpha)|}{\alpha} \sup \{|z-1|: z \in W(S, T)\},
$$

which offers a practical way to estimate the spectral distance when it is small. The spectral distance serves both as a means to quantify the quality of a preconditioner and also as a convenient analysis tool for multigrid algorithms. Essentially, if two operators $S, T$ satisfy

$$
1-\delta \leqslant\left|\frac{\left\langle S_{\mathbb{C}} w, w\right\rangle}{\left\langle T_{\mathbb{C}} w, w\right\rangle}\right| \leqslant 1+\delta, \quad \forall w \in \mathcal{X}^{\mathbb{C}} \backslash\{0\},
$$

with $\delta \ll 1$, then $d_{\sigma}(S, T) \approx \delta$. If $N \approx G^{-1}$ is a preconditioner for $G$, then both $d_{\sigma}\left(N, G^{-1}\right)$ and $d_{\sigma}\left(N^{-1}, G\right)$ (quantities which are are equal if $G, N$ are symmetric) are shown to control the spectral radius $\rho(I-N G$ ) (see Lemma A.2 in Appendix Afor a precise formulation), which is an accepted quality-measure for a preconditioner. The advantage of using $d_{\sigma}$ over $\rho(I-N G)$ is that the former is a true distance function. 
THEOREm 4.9. If the operators $\mathcal{K}, \mathcal{K}_{h}$ satisfy Condition 2.1 on the locallysymmetric meshes $\mathcal{T}_{h}$ with the weights given by (2.1), and $\lambda \in W_{\infty}^{2}(\Omega)$ satisfies $\mathcal{I}_{h} \lambda=\lambda_{h}$, there exist $C, \delta>0$ independent of $h, \lambda$ so that for $h^{2}\left\|\lambda^{-\frac{1}{2}}\right\|_{W_{\infty}^{2}(\Omega)} \leqslant \delta$

$$
d_{\sigma}\left(\mathcal{G}_{h}^{-1}, \mathcal{S}_{h}\right) \leqslant C h^{2}\left\|\lambda^{-\frac{1}{2}}\right\|_{W_{\infty}^{2}(\Omega)},
$$

where $\mathcal{G}_{h}$ and $\mathcal{S}_{h}$ are defined as in (4.4) and (4.8). If the meshes are not locallysymmetric then the power of $h$ in (4.29) is 1 .

Proof. Again, we restrict our attention to the locally-symmetric case. The operator $\mathcal{G}_{h}$ is symmetric positive definite with respect $\langle\cdot, \cdot\rangle_{h}$ and satisfies

$$
\left\langle\mathcal{G}_{h} u, u\right\rangle_{h}=\|u\|_{h}^{2}+\left\|\mathcal{L}_{h} u\right\|_{h}^{2} \geqslant\|u\|_{h}^{2} .
$$

Therefore $\sigma\left(\mathcal{G}_{h}\right) \subseteq[1, \infty)$, and $\left\|\mathcal{G}_{h}^{\nu}\right\|_{h} \leqslant 1$ for all $\nu<0$. Due to the norm equivalence $\|\cdot\|\left\|_{h} \sim\right\| \cdot \|$ there exists $C_{1}>0$ so that $\left\|\mathcal{G}_{h}^{-\frac{1}{2}}\right\| \leqslant C_{1}$. By Lemma 4.2 and Propositions 4.5 and 4.8 we have

$$
\left\|\mathcal{G}_{h}-\mathcal{N}_{h}\right\| \leqslant C_{2} h^{2}\left\|\lambda^{-\frac{1}{2}}\right\|_{W_{\infty}^{2}(\Omega)}
$$

for some constant $C_{2}>0$. Hence for $C_{3}=C_{1}^{2} C_{2}$

$$
\left\|I-\mathcal{G}_{h}^{-\frac{1}{2}} \mathcal{N}_{h} \mathcal{G}_{h}^{-\frac{1}{2}}\right\| \leqslant\left\|\mathcal{G}_{h}^{-\frac{1}{2}}\right\|^{2} \cdot\left\|\mathcal{G}_{h}-\mathcal{N}_{h}\right\| \leqslant C_{3} h^{2}\left\|\lambda^{-\frac{1}{2}}\right\|_{W_{\infty}^{2}(\Omega)} .
$$

Since for any operator $T$ we have $\left\|I-T^{-1}\right\| \leqslant(1-\alpha)^{-1}\|I-T\|$ if $\|I-T\| \leqslant \alpha$,

$$
\left\|I-\mathcal{G}_{h}^{\frac{1}{2}} \mathcal{S}_{h} \mathcal{G}_{h}^{\frac{1}{2}}\right\| \stackrel{\mathcal{S}_{h}=\mathcal{N}_{h}^{-1}}{\leqslant} \frac{4}{3}\left\|I-\mathcal{G}_{h}^{-\frac{1}{2}} \mathcal{N}_{h} \mathcal{G}_{h}^{-\frac{1}{2}}\right\| \leqslant \frac{4 C_{3}}{3} h^{2}\left\|\lambda^{-\frac{1}{2}}\right\|_{W_{\infty}^{2}(\Omega)}
$$

provided $h^{2}\left\|\lambda^{-\frac{1}{2}}\right\|_{W_{\infty}^{2}(\Omega)} \leqslant \delta=\frac{1}{4 C_{3}}$. By further restricting $\delta$, we can assume the righthand side of (4.30) to be $\leqslant 1 / 2$, which implies that $W\left(\mathcal{S}_{h}, \mathcal{G}_{h}^{-1}\right) \subset \mathcal{B}_{\frac{1}{2}}(1)$. By (4.28) we obtain

$$
d_{\sigma}\left(\mathcal{S}_{h}, \mathcal{G}_{h}^{-1}\right) \leqslant \frac{8 \ln 2}{3} C_{3} h^{2}\left\|\lambda^{-\frac{1}{2}}\right\|_{W_{\infty}^{2}(\Omega)} .
$$

The highlight of the last result is the presence of $O\left(h^{2}\right)$ (or $O(h)$ for general quasi-uniform meshes) in the right-hand side of (4.29), which is the optimal order of approximation in $h$. We should stress that for classical multigrid methods for differential equations one has $O(1)$ as the right-hand side estimate, which is sufficient for mesh-independence. In this case, if the theoretically introduced smooth function $\lambda$ could be the same for all meshes, the number of $\mathcal{S}_{h}$-preconditioned iterations is expected to decrease with $h \downarrow 0$. In reality, the discrete function $\lambda_{h}$ is tied to the Lagrange multipliers $\mathbf{v}_{1}, \mathbf{v}_{2}$, which in turn are related (actually expected to converge to as $\mu, h \downarrow 0$ ) the Lagrange multipliers $\underline{\lambda}, \bar{\lambda}$ of the continuous problem. Since in general the latter are only in $L^{2}$, the factor $\left\|\lambda^{-\frac{1}{2}}\right\|_{W_{\infty}^{2}(\Omega)}$ is expected to be unbounded as $\mu \downarrow 0$. Therefore the preconditioning qualities of $\mathcal{S}_{h}$ are expected to increase with $h \downarrow 0$, but decrease with $\mu \downarrow 0$. Thus for large-scale, high-resolution problems, where $h \ll 1$, the presented method is expected to perform very well, especially in connection with the multigrid method discussed in the next section. However, for fixed $h$, as $\mu \downarrow 0$ in the IPM formulation and the approximate solution approaches $\widehat{\mathbf{u}}$, if the inequality constraints are active then the quality of the proposed preconditioner normally degrades. The advantages or disadvantages of this method will ultimately be discussed based on numerical experiments in Section 6 . 
5. The multigrid preconditioner. While the two-grid preconditioner $\mathcal{S}_{h}$ may be efficient in terms of number of iterations, it is expensive to apply. In this section we develop a multigrid preconditioner $\mathcal{S}_{h}^{m g}$ that also satisfies the optimal order estimate (4.29) but has a lesser cost. Since the process of passing from a two-grid to a multigrid preconditioner of comparable quality has been analyzed in 13, we give here only a brief description. In this section we assume a finite number of grids

$$
I_{\max }=\left\{h_{i}\right\}_{0 \leqslant i \leqslant i_{\max }}, h_{i}=h_{0} 2^{-i},
$$

and the goal is to ultimately construct an efficient multigrid preconditioner for the operator on the finest grid.

Consider the operator $\mathfrak{I}_{i-1}^{i}: \mathfrak{L}\left(\mathcal{V}_{h_{i-1}}\right) \rightarrow \mathfrak{L}\left(\mathcal{V}_{h_{i}}\right)$ by

$$
\mathfrak{I}_{i-1}^{i}(\mathcal{M}) \stackrel{\text { def }}{=}\left(I-\pi_{h_{i-1}}\right)+\mathcal{M} \pi_{h_{i-1}} \text {. }
$$

Cf. (4.8) we have $\mathcal{S}_{h_{i}}=\mathfrak{I}_{i-1}^{i}\left(\mathcal{G}_{h_{i-1}}^{-1}\right)$. If we define $\mathcal{S}_{h}^{V}, h \in I_{\max }$, recursively by

$$
\mathcal{S}_{h_{i}}^{V}= \begin{cases}\mathcal{G}_{h_{0}}^{-1} & \text { if } \quad i=0, \\ \mathfrak{I}_{i-1}^{i}\left(\mathcal{S}_{h_{i-1}}^{V}\right) & \text { if } \quad 1 \leqslant i \leqslant i_{\max }\end{cases}
$$

then $\mathcal{S}_{h}^{V}$ has a V-cycle structure. However, it is shown in [13] that $\mathcal{S}_{h}^{V}$ is suboptimal, in that it satisfies (4.29) with $h^{2}$ replaced by $h_{0}^{2}$. Thus the quality of $\mathcal{S}_{h}^{V}$ does not improve with $h \downarrow 0$, as desired, it is simply mesh-independent (it only depends on $h_{0}$ ). To achieve the desired result we define the operator $\mathfrak{N}_{i}: \mathfrak{L}\left(\mathcal{V}_{h_{i}}\right) \rightarrow \mathfrak{L}\left(\mathcal{V}_{h_{i}}\right)$

$$
\mathfrak{N}_{i}(\mathcal{M}) \stackrel{\text { def }}{=} 2 \mathcal{M}-\mathcal{M} \mathcal{G}_{h_{i}} \mathcal{M}
$$

The latter is related to Newton's method for the operator-equation $\mathcal{X}^{-1}-\mathcal{G}_{h_{i}}=0$; namely, if $\mathcal{X}_{0}$ is a good guess at the solution, i.e., approximates well $\mathcal{G}_{h_{i}}^{-1}$, then the first Newton iterate starting at $\mathcal{X}_{0}$ is $\mathcal{X}_{1}=\mathfrak{N}_{i}\left(\mathcal{X}_{0}\right)$ (see also Remark 3.11 in [13]). We define the multigrid preconditioner using the following algorithm:

Algorithm 1: Operator-form definition of $\mathcal{S}_{h_{i}}^{m g}$

1. if $i=0$

2. $\mathcal{S}_{h_{0}}^{m g}:=\mathcal{G}_{h_{0}}^{-1}$

$\%$ coarsest level

3. else if $i<i_{\max }$

4. $\quad \mathcal{S}_{h_{i}}^{m g}:=\mathfrak{N}_{i}\left(\mathfrak{I}_{i-1}^{i}\left(\mathcal{S}_{h_{i-1}}^{m g}\right)\right) \quad \%$ intermediate level

5. else

6. $\quad \mathcal{S}_{h_{i}}^{m g}:=\mathfrak{I}_{i-1}^{i}\left(\mathcal{S}_{h_{i-1}}^{m g}\right) \quad \%$ finest level

7. end if

8. end if

The key factor in Algorithm 1 is the application of $\mathfrak{N}_{i}$ at Step 4, and here is why: while $\mathcal{G}_{h_{i}}^{-1}$ is well approximated by $\mathfrak{I}_{i-1}^{i}\left(\mathcal{S}_{h_{i-1}}^{m g}\right.$ ) provided that $\mathcal{G}_{h_{i-1}}^{-1} \approx \mathcal{S}_{h_{i-1}}^{m g}$ (recall that $\left.\mathcal{G}_{h_{i}}^{-1} \approx \mathcal{S}_{h_{i}}=\Im\left(\mathcal{G}_{h_{i-1}}^{-1}\right)\right)$, an application of $\mathfrak{N}_{i}$ brings $\mathfrak{I}\left(\mathcal{S}_{h_{i-1}}^{m g}\right)$ even closer to $\mathcal{G}_{h_{i}}^{-1}$. This step is critical if we want $d_{\sigma}\left(\mathcal{G}_{h}^{-1}, \mathcal{S}_{h}^{m g}\right)=O\left(d_{\sigma}\left(\mathcal{G}_{h}^{-1}, \mathcal{S}_{h}\right)\right)$. Also, there are two 
main reasons for splitting the cases of intermediate vs. finest resolution, as opposed to just replacing $\mathfrak{I}_{i-1}^{i}\left(\mathcal{S}_{h_{i-1}}^{V}\right)$ with $\mathfrak{N}_{i}\left(\mathfrak{I}_{i-1}^{i}\left(\mathcal{S}_{h_{i-1}}^{V}\right)\right)$ in (5.1). First we would like to have $\mathcal{S}_{h}^{m g}=\mathcal{S}_{h}$ for $h=h_{i_{\max }}$ if only two grids are used. Second, the application of $\mathfrak{N}_{i}$ includes a multiplication by $\mathcal{G}_{h_{i}}$; since for the intended large-scale applications the finest-level mat-vec is expected to be very costly, we prefer that no such mat-vecs are computed inside the preconditioner.

THEOREM 5.1. In the hypotheses of Theorem 4.9, and with $\mathcal{S}_{h}^{m g}$ defined as in Algorithm 1 there exist $C, \delta>0$ independent of $h$ and $\lambda$ so that for $h^{2}\left\|\lambda^{-\frac{1}{2}}\right\|_{W_{\infty}^{2}(\Omega)} \leqslant \delta$

$$
d_{\sigma}\left(\mathcal{G}_{h}^{-1}, \mathcal{S}_{h}^{m g}\right) \leqslant C h^{2}\left\|\lambda^{-\frac{1}{2}}\right\|_{W_{\infty}^{2}(\Omega)}, \text { for } h=h_{\max } .
$$

The proof of Theorem 5.1 follows closely that of Theorem 5.4 in [13] and, in the interest of brevity, we do not give further details. Suffice it to say that the use of the spectral distance is instrumental, and that an essential ingredient is the symmetry (with respect to $\langle\cdot, \cdot\rangle_{h}$ ) of $\mathcal{G}_{h}$.

In practice, for large-scale problems, neither $\mathcal{G}_{h}$ nor $\mathcal{S}_{h}^{m g}$ are ever formed, so both are to be applied matrix-free. A simple verification shows that, given some $\mathcal{M} \in \mathfrak{L}\left(\mathcal{V}_{h_{i}}\right)$, the vector $\tilde{u}=\left(\mathfrak{N}_{i}(\mathcal{M})\right) r$ can be computed by setting $\tilde{u}:=u_{2}$ where $u_{k+1}:=u_{k}+\mathcal{M}\left(r-\mathcal{G}_{h_{i}} u_{k}\right)$ with $u_{0}=0$. Thus the matrix-free application of $\mathcal{S}_{h}^{m g}$ is computed by the following function:

Algorithm 2: Matrix-free implementation of the action $u=\mathcal{S}_{h}^{m g} r$.

1. function $u=M G(r, i)$

2. if $i=0$

$\%$ coarsest level

3. $u:=\mathcal{G}_{h_{0}}^{-1} r$ $\%$ direct or unpreconditioned CG solve

4. else

5. $\quad u:=\left(I-\pi_{h_{i-1}}\right) r+M G\left(\pi_{h_{i-1}} r, i-1\right)$

6. if $i<i_{\max } \quad \%$ intermediate level

7. $\quad r_{1}:=r-\mathcal{G}_{h_{i}} u$

8. $\quad u_{1}:=\left(I-\pi_{h_{i-1}}\right) r_{1}+M G\left(\pi_{h_{i-1}} r_{1}, i-1\right)$

9. $\quad u:=u+u_{1}$

10. end if

11. end if

As can be readily seen, Algorithm 2 has a $\mathrm{W}$-cycle structure. To estimate the cost of $M G\left(\cdot, i_{\max }\right)$ we denote by $T(i)$ the cost of applying $M G(\cdot, i)$ for $0<i<i_{\max }$. If we assume that one residual computation at level $i$ has complexity $O\left(N_{h_{i}}^{2}\right)$, and that the cost of computing an $L^{2}$-projection is negligible compared to that of a residual computation (this is reasonable for most applications since mass matrices are normally easy to invert) then the resulting recursion for the function $T$ reads:

$$
T(i)=O\left(N_{h_{i}}^{2}\right)+2 T(i-1) .
$$

For $i=i_{\text {max }}$ the term $O\left(N_{h_{i}}^{2}\right)$ is replaced by a potentially smaller cost of just computing an $L^{2}$-projection. Given that $N_{h_{i-1}} \approx 2^{-d} N_{h_{i}}$ (here $d=2$ ), a standard argument 
shows that

$$
T\left(i_{\max }\right)=O\left(N_{i_{\max }}^{2}\right)
$$

that is, a cost that is proportional to that of a residual computation.

Another comment refers to a detail that is not very transparent in Algorithm 2, namely that coarse versions of $\lambda_{h_{i}}$ are necessary for each level, because

$$
\mathcal{G}_{h_{i}}=I+\left(\mathcal{D}_{1 / \sqrt{\lambda_{h_{i}}}}^{h_{i}}\right)^{*}\left(\mathcal{K}_{h_{i}}\right)^{*} \mathcal{K}_{h_{i}} \mathcal{D}_{1 / \sqrt{\lambda_{h_{i}}}}^{h_{i}}
$$

Since the original problem is solved starting at the finest level, where $\lambda_{h_{i_{\max }}}$ is given by the optimization algorithm, the functions $\lambda_{h_{i}}$ are obtained by simply discarding the values at finer nodes of $\lambda_{h_{i_{\max }}}$. The parameter $\beta$ is hidden in $\lambda$ and affects the process indirectly.

6. Applications and numerical examples. In this section we discuss two applications. The first is related to the inverse contamination problem studied in [2, 1, where $\mathcal{K}$ is a time- $T$ solution operator of a parabolic equation. The second is a standard elliptic-constrained optimal control problem with additional box-constraints on the control.

6.1. Solution strategies and metrics for success. For both applications we apply Mehrotra's algorithm and we solve the inner linear systems using the multigrid preconditioner previously defined. In the absence of multiple grids, the linear systems (3.12) are solved using conjugate gradient (CG), while for more than one level we used MG-preconditioned conjugate gradient squared (CGS), because of the slight non-symmetry of the MG preconditioner. As a first metric we record the number of inner linear iterations needed at each outer iteration; secondly, we record the total number of finest-grid mat-vecs for the entire solution process. Recall that each outer iteration requires the solution of two linear systems with identical matrices, namely one for the predictor step and one for the corrector step; in the interest of the presentation we record only the linear iterations for the predictor step. Also, a small number of mat-vecs are required in the process in addition to those needed for the predictor-corrector solves, and are reflected in the count. With respect to the second metric we remark that the proposed algorithm is intended for large-scale problems, with the most expensive computation being the finest-scale residual computation. The ultimate goal is to significantly reduce the total number of finest-scale mat-vecs, because this is expected to be directly linked with execution time in a truly largescale computation. With regard to the second metric we would like the total number of finest-level mat-vecs to decrease with $h \downarrow 0$. As for the first metric (number of iterations) we would like to witness the following:

[a.] The number of MG-preconditioned CGS-iterations should be less than half of the unpreconditioned CG-iterations (each CGS-iteration involves two mat-vecs, while a $\mathrm{CG}$ iteration requires only one).

[b.] For a given resolution $h$, the number of MG-preconditioned CGS iterations should be relatively bounded with respect to the number of levels used, provided the coarsest level is sufficiently fine, as stated in Theorem 5.1 .

[c.] Mostly important, the number of MG-preconditioned CGS iterations should decrease with $h \downarrow 0$; in other words, the MG-preconditioned CGS becomes increasingly advantageous compared to CG as the problem-size increases. One word of caution though: linear systems of different resolutions are not necessarily related in 
a direct fashion, since their " $\lambda$ " is dictated by the evolution of Mehrotra's algorithm, which is slightly different for each resolution. For example, the tenth linear system to be solved in the IPM process at a resolution $2 h$ is not necessarily some coarse version of the tenth system to be solved at resolution $h$.

Also, we should point out that in all our tests we use a cold start, that is, we do not take advantage of results from coarser levels except for in the MG-solve of the inner linear systems. While for realistic applications "warm-start" strategies are essential, we restrict our attention to the way our multigrid technology plays a role in solving the inner linear systems.

6.2. Time-reversal for a parabolic equation. We consider the problem of finding the initial state (the control) for a system governed by a parabolic equation given the state at a later time $T$ under additional box-constraints on the control. Multigrid-preconditioning for the unconstrained version of this problem was studied in detail in [12, 13] and for the space-time measurements in [2].

Formally, we consider the following parabolic initial value problem with periodic boundary conditions

$$
\begin{cases}\partial_{t} y-\partial_{x}\left(a \partial_{x} y+b y\right)+c y=0 & , \quad \text { on }[0,1] \times(0, T], \\ y(0, t)=y(1, t), \partial_{x} y(0, t)=\partial_{x} y(1, t) & , \quad \text { for } t \in(0, T] \\ y(x, 0)=u(x) & , \quad \text { for } x \in[0,1]\end{cases}
$$

where $a>0, b, c \geqslant 0$ are constants, and $T>0$ is the end-time. For $t>0$ we denote by $\mathcal{S}(t) \in \mathfrak{L}\left(L^{2}([0,1])\right)$ the time- $t$ solution operator mapping the initial value onto $y(\cdot, t)$

$$
u \stackrel{\mathcal{S}(t)}{\longmapsto} y(\cdot, t)
$$

and let $\mathcal{K}=\mathcal{S}(T)$. The discrete $\mathcal{K}_{h}$ is obtained by using a Galerkin formulation with continuous piecewise elements on a uniform grid for the spatial discretization and Crank-Nicolson in time. It is shown in [30] (see also [36]) that for sufficiently small $h$ the following estimate holds:

$$
\left\|\mathcal{K} u-\mathcal{K}_{h} \pi_{h} u\right\|_{H^{m}} \leqslant C h^{2-m}\|u\|, \forall u \in L^{2}([0,1]), m=0,1,
$$

where $C=C(T)$, provided the time step $k$ is proportional to the spatial resolution $k=C_{1} h$, with $C_{1}$ chosen to ensure stability. Consequently, space and time resolutions are refined at the same rate, and Condition 2.1 is verified, so our theory applies. The specific details (boundary conditions, constant advection etc.) in this example were chosen for convenience, however, two and three spatial dimensions, other types of boundary conditions, as well as smoothly varying functions in place of the constants $a, b, c$ are supported.

A direct verification of the convergence order. As mentioned earlier, when running Mehrotra's algorithm with different resolutions, the added diagonal terms $\lambda$ may not be in direct relationship with each other. Hence, in order to practically verify the presence of $h^{2}$ in the estimate (4.29) we resort to an artificial context: we construct $\mathcal{G}_{h}$ based on a fixed function $\lambda_{h}=\mathcal{I}_{h}(\sin )+\beta$ for $h_{j}=80 \cdot 2^{j}, j=0,1,2,3$, and we define the corresponding two-grid preconditioners $\mathcal{N}_{h}$. Then we compute the "distances" $d_{h}=\max \left\{|\ln \alpha|: \alpha \in \sigma\left(\mathcal{G}_{h}, \mathcal{N}_{h}\right)\right\}$. Since $d_{h}$ approximates the spectral distance of interest (because $\mathcal{N}_{h}$ is close to being symmetric, actually we have $d_{h} \leqslant d_{\sigma}$ ) we expect to see that $d_{h}=O\left(h^{2}\right)$. We repeat the experiment for $\beta=1,0.1,0.01$. The results presented in Table 6.1, while not yet converged, give a strong indication of an asymptotic rate $\lim _{h \rightarrow 0} d_{2 h} / d_{h}=4$. 
TABLE 6.1

$$
d_{h}=\max \left\{|\ln \alpha|: \alpha \in \sigma\left(\mathcal{G}_{h}, \mathcal{N}_{h}\right)\right\} \text { for } \lambda(x) \approx(\sin (x)+\beta) .
$$

\begin{tabular}{|l|l|l|l|l|l|l|}
\hline$h \backslash \beta$ & \multicolumn{2}{|c|}{1} & \multicolumn{2}{c|}{0.1} & \multicolumn{2}{c|}{0.01} \\
\hline & $d_{h}$ & rate & $d_{h}$ & rate & $d_{h}$ & rate \\
\hline $1 / 80$ & 0.0206 & & 0.1127 & & 0.2812 & \\
$1 / 160$ & 0.0066 & 3.1342 & 0.0363 & 3.1078 & 0.1270 & 2.2140 \\
$1 / 320$ & 0.0020 & 3.3140 & 0.0102 & 3.5488 & 0.0445 & 2.8535 \\
$1 / 640$ & 0.0006 & 3.5199 & 0.0027 & 3.7365 & 0.0123 & 3.6284 \\
\hline
\end{tabular}

Numerical study. We consider the "true" initial value $u_{0}$ supported on two intervals with $u_{0}$ reaching the value 1 on one of the intervals and $1 / 2$ on the other interval, then let $f=\mathcal{K} u_{0}$. Specific values are $a=4 \cdot 10^{-3}, b=0.4, c=0$, and $T=0.8$. In Figure 6.1 we show $u_{0}, f$ as well as the converged solution $u_{\min }$ of the box-constrained optimization problem with $\beta=10^{-3}$, a value chosen because of the relatively good (visual) agreement of $u_{0}$ with $u_{\min }$. We run Mehrotra's algorithm for $h=2^{-10}, 2^{-11}, 2^{-12}, 2^{-13}$, and for each $h$ we test the solvers with 1,2 , and 3 levels. The number of linear iterations required by each of the linear solves in the predictor step are shown in Figure 6.2, while the corresponding values for $\left\|\lambda^{-1 / 2}\right\|_{W_{\infty}^{2}}$ and $\mu$ are shown in the top two pictures of Figure 6.3

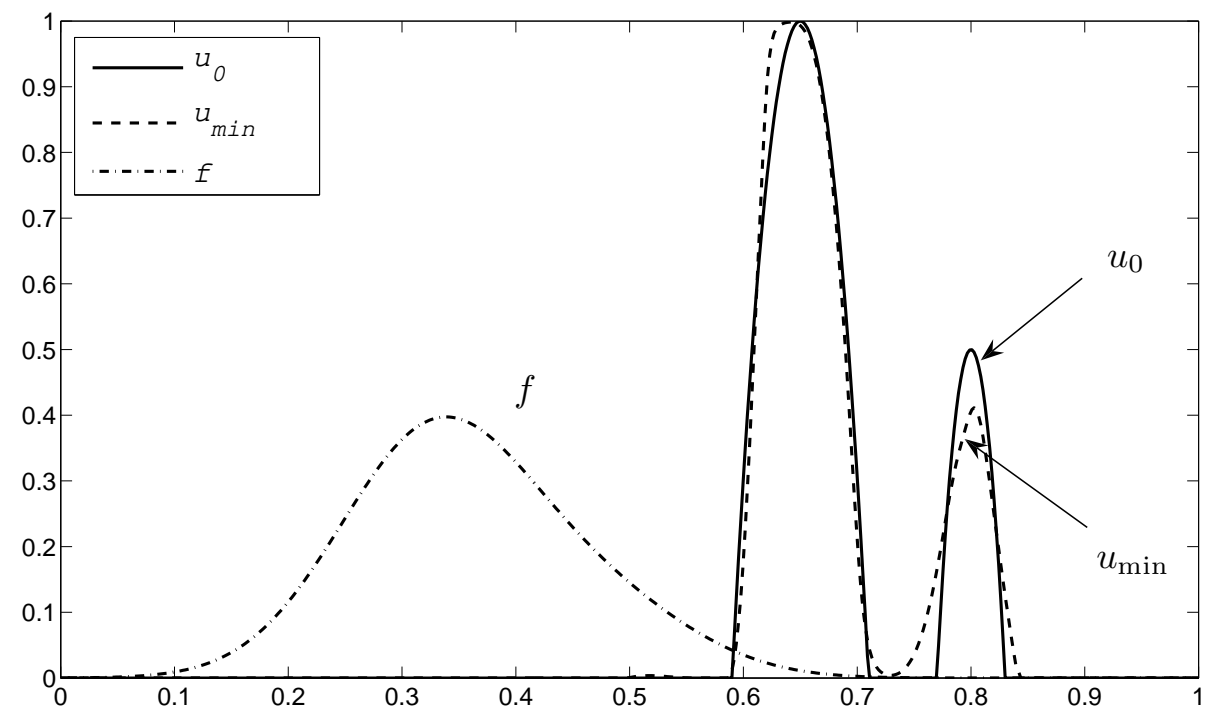

FIG. 6.1. Solution with $f=\mathcal{K} u_{0}, \beta=10^{-3}, 0 \leqslant u \leqslant 1$.

First we remark that the number of unpreconditioned CG iterations appears to be mesh-independent (top chart in Figure 6.2): essentially the curves representing the number of iterations for each of the resolutions more-or-less overlap. We notice only a slight increase in number of iterations for higher resolutions. Second, from the middle chart in Figure 6.2 representing the number of two-grid CGS iterations, we infer that the number of two-grid preconditioned iterations consistently decreases 


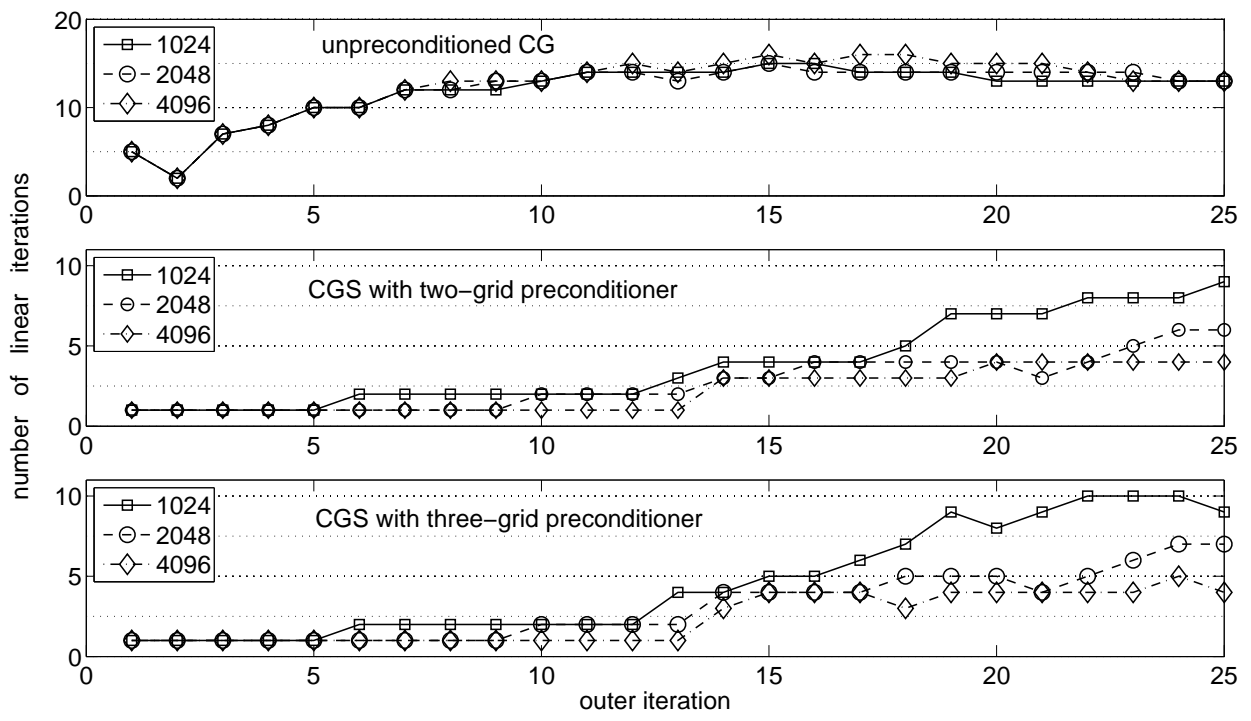

FIG. 6.2. Number of iterations for each of the predictor-step linear systems solved $\left(\beta=10^{-3}\right)$.
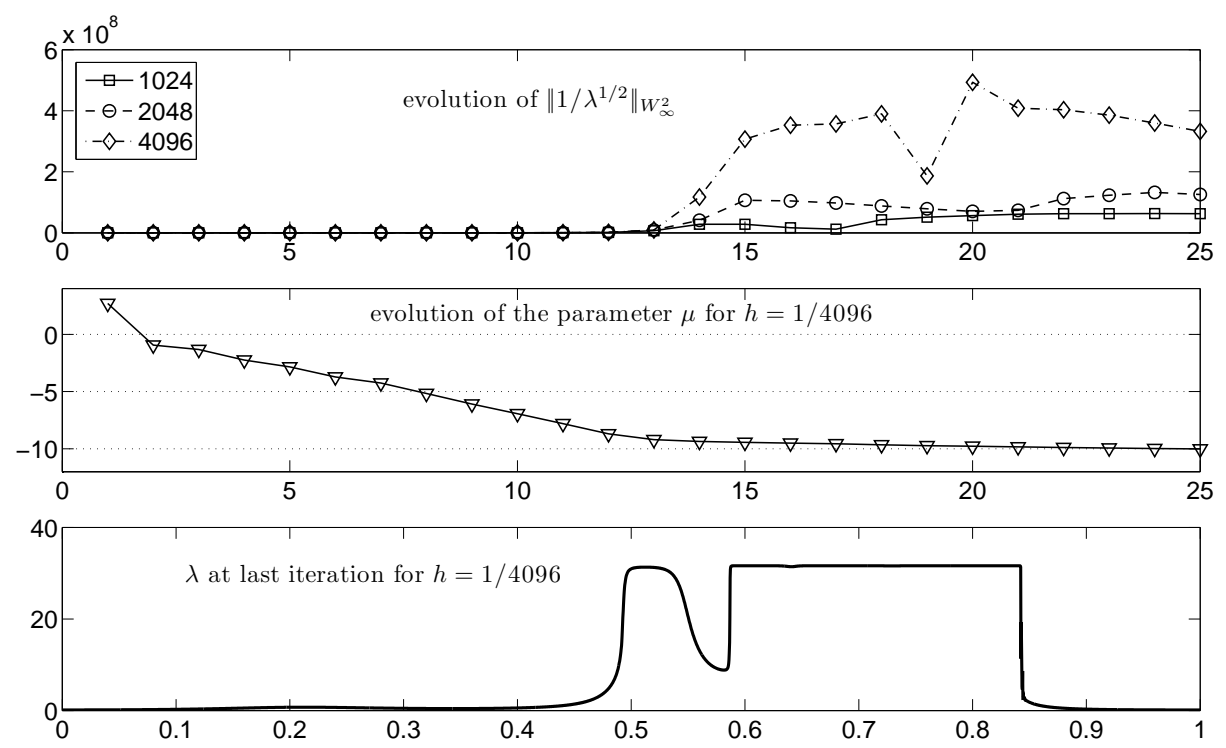

FIG. 6.3. Top: discrete $\left\|\lambda_{h}^{-\frac{1}{2}}\right\|_{W_{\infty}^{2}}$ based on discrete Laplacian should give an idea of the size of $\left\|\lambda^{-\frac{1}{2}}\right\|_{W_{\infty}^{2}}$; middle: log-plot of $\mu$ as a function of outer iteration; bottom: $\lambda_{h}$ for $h=1 / 4096$ at the last outer iterate $\left(\beta=10^{-3}\right)$.

with $h \downarrow 0$, as desired. For example, at the twenty-fourth iteration these numbers are 8,6 , and 4 , while at the twentieth they are $7,4,4$. This phenomenon is repeated for the three-grid preconditioner as can be seen from the bottom chart in Figure 6.2. 
Moreover, for the most part, the number of MG-CGS preconditioned iterations is significantly smaller than half the number of unpreconditioned CG iterations: e.g., for $h=1 / 4096$, up to the thirteenth iteration (where $\mu$ is already down to approx. $10^{-8}$, see Figure 6.3) only one two-grid (or three-grid) MG-preconditioned CGS iteration is necessary, while up to 15 unpreconditioned CG iterations are needed. However, after the thirteenth iteration $\left\|\lambda^{-1 / 2}\right\|_{W_{\infty}^{2}}$ shows a significant increase, as seen on the top chart of Figure 6.3 and the MG-preconditioned CGS is less efficient: for $h=1 / 4096$, at the twenty-fourth iteration 4 two-grid iterations are needed compared to $13 \mathrm{CG}$ iterations, a lesser advantage compared to the earlier outer iterations. The bottom chart in Figure 6.3 shows the last computed $\lambda_{h}$ at $h=1 / 4096$ to give an idea of why the quantity $\left\|\lambda^{-1 / 2}\right\|_{W_{\infty}^{2}}$ is so large. A comparison between the bottom and middle charts in Figure 6.2 shows that the number of MG-preconditioned iterations is not very sensitive to the number of levels, provided the coarsest mesh is sufficiently fine. In this example four levels would force a much too coarse base mesh, and produce unsatisfactory preconditioners. The last piece of evidence is the total count of finestlevel mat-vecs, shown in Table 6.2. In this example, a mat-vec involves solving the advection-reaction-diffusion equation on $[0, T]$. The data clearly shows that, as $h \downarrow 0$, the two-level solvers is getting increasingly efficient in this metric compared to CG: the ratio goes from $581 / 728$ for $h=1 / 1024$ to $377 / 768$ for $h=1 / 8192=2^{-13}$. We should remark also that the essential impediment to a more significant improvement over CG lies in the increase in the $\left\|\lambda^{-1 / 2}\right\|_{W_{\infty}^{2}}$ as $h \downarrow 0$. As shown in Theorem 4.9, non-smoothness of $\lambda^{-1 / 2}$ decreases the preconditioner's efficiency.

TABLE 6.2

Total number of fine-grid mat-vecs for the $1 \mathrm{D}$ reversed parabolic equation

\begin{tabular}{|l|l|l|l|}
\hline$h \backslash$ levels & 1 & 2 & 3 \\
\hline $1 / 1024$ & 728 & 581 & 661 \\
$1 / 2048$ & 740 & 463 & 489 \\
$1 / 4096$ & 764 & 403 & 425 \\
$1 / 8192$ & 768 & 377 & 403 \\
\hline
\end{tabular}

TABLE 6.3 Total number of fine-grid mat-vecs for the 2D elliptic-constrained opt. ctrl. problem

\begin{tabular}{|l|l|l|l|l|}
\hline$h \backslash$ levels & 1 & 2 & 3 & 4 \\
\hline $1 / 256$ & 354 & 282 & 572 & - \\
$1 / 512$ & 355 & 220 & 250 & 452 \\
$1 / 1024$ & 355 & 198 & 210 & 224 \\
$1 / 2048$ & 363 & 172 & 174 & 174 \\
\hline
\end{tabular}

6.3. An elliptic-constrained control problem. In this example we discuss the elliptic-constrained optimal control problem (1.4) from Example B, which is a standard test problem in PDE-constrained optimization [8, 26], and corresponds to (1.1) with $\mathcal{K}=\Delta^{-1}$. We consider a square domain with a continuous piecewise linear finite element discretization based on the standard three-lines triangular mesh 2 . Standard estimates for finite element solutions of elliptic problems show that Condition 2.1 is verified [9].

Numerical study. Let $\Omega=[0,1] \times[0,1], \beta=10^{-6}$, and $f$ be the function that satisfies $\Delta f=u_{0},\left.f\right|_{\partial \Omega}=0$, where $u_{0}(x, y)=\frac{3}{2} \sin (2 \pi x) \sin (2 \pi y)$. With this selection of $f$, the choice $u=u_{0}$ would be a solution of (1.4) if $\beta=0$ and no box constraints were present (or if $\left[-\frac{3}{2}, \frac{3}{2}\right] \subseteq[\underline{u}, \bar{u}]$ ). Here the bounds $[\underline{u}, \bar{u}]=[-1,1]$ are active: without them, given that $\beta \ll 1$, the solution would be close to $u_{0}$, that is, would have a maximum (resp. minimum) close to $3 / 2$ (resp. $-3 / 2$ ). The solution with $h=1 / 128$ is depicted in Figure 6.4. We have solved the problem with $h=2^{-8}, 2^{-9}, 2^{-10}, 2^{-11}$

\footnotetext{
${ }^{2}$ The three-line mesh is obtained by dividing the square into equally sized squares with sides parallel to the coordinate axes, and by further cutting each little square along its slope-one diagonal.
} 
using one, two, three, and four levels (where appropriate) using the strategy described in Section 6.1

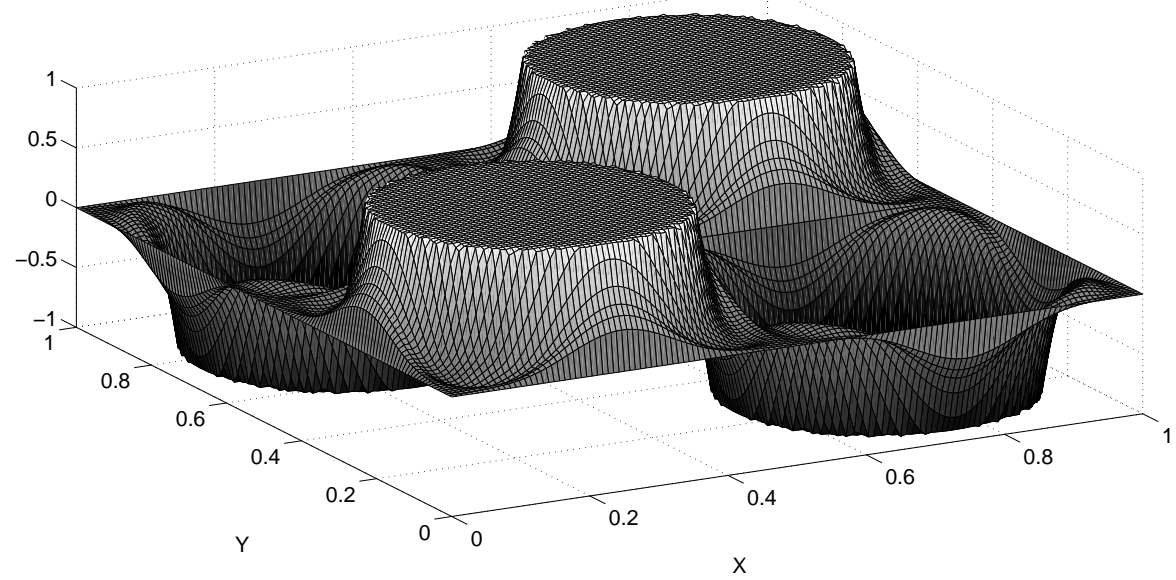

FIG. 6.4. Solution with $f$ satisfying $\Delta f=\frac{3}{2} \sin (2 \pi x) \sin (2 \pi y), \beta=10^{-6},[\underline{u}, \bar{u}]=[-1,1]$.

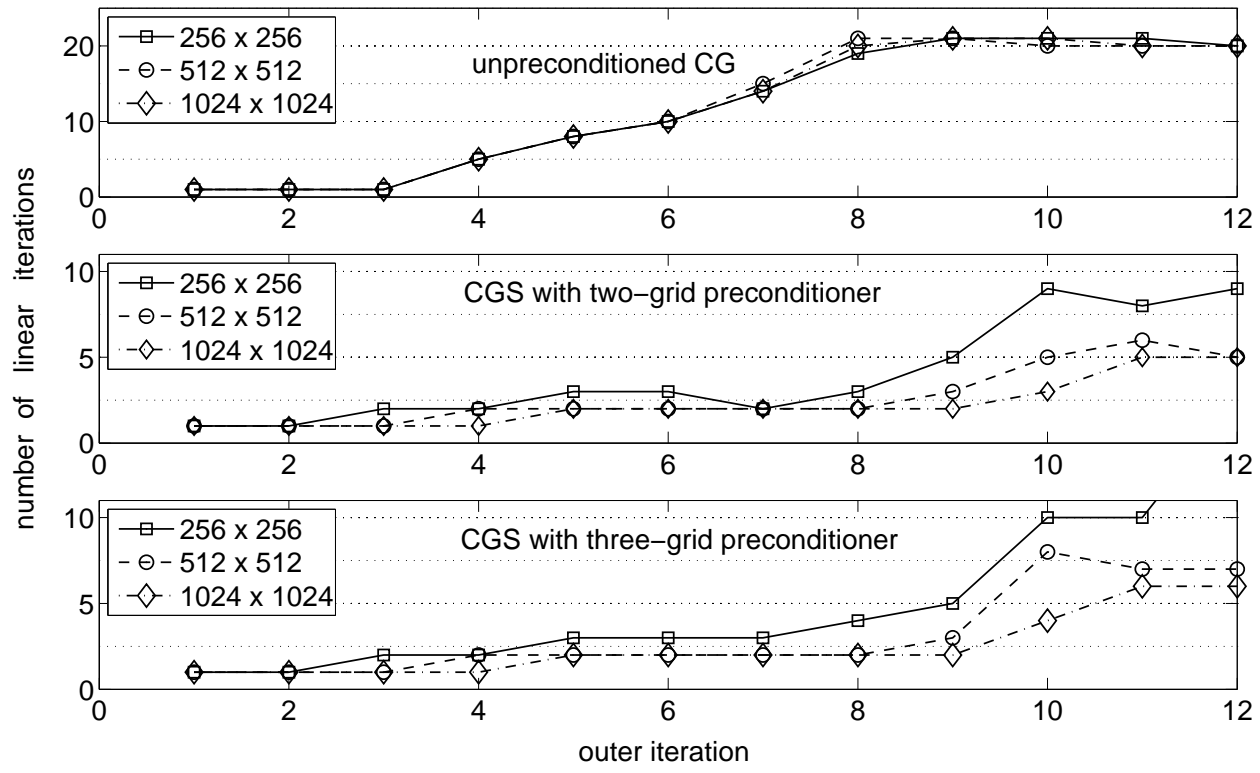

FIG. 6.5. Number of iterations for each of the predictor-step linear systems solved $\left(\beta=10^{-6}\right)$.

As with the previous example, we show in Figure 6.5 the number of iterations required by each of the linear systems at the predictor step. The top plot shows the number of unpreconditioned iterations to level off at 21. The middle plot again shows two facts: the number of MG-preconditioned CGS iterations decreases with $h \downarrow 0$. In addition, for the finest grid, the number of two-grid preconditioned CGS 
iterations is less than $1 / 4$ that of the number of iterations for the unpreconditioned case even when looking beyond the eighth outer iteration, where as before, roughness of the $\lambda$-function lowers the quality of the MG-algorithm. For example, for the tenth outer iteration with $h=1 / 1024$, CGS required 3 iterations, while 21 iterations were needed in the unpreconditioned case; for the eleventh outer iteration the numbers are 5 vs. 20. Of course, each two-grid preconditioned CGS iteration is significantly more expensive than an unpreconditioned CG iteration, which is why the number of levels should be maximized. The bottom plot in Figure 6.5 shows that the two-level behavior is replicated using three-level preconditioners. Moreover, at fine resolutions $\left(h \leqslant 2^{-10}\right)$, the numbers of required three-level preconditioned CGS iterations are not significantly higher than those of two-level CGS iterations. However, this is not the case for low resolution $h=2^{-8}$, where the number of three-level CGS iterations required for the last three systems (not shown on the plot) is quite large: 45, 17, 58 . This is why we insist that the MG-preconditioner is efficient only when the coarsest resolution used is sufficiently fine and the finest resolution $h$ is small. With respect to the second metric, we show in Table 6.3 the total number of fine mat-vecs for each of the runs, and the results confirm that the MG-preconditioner becomes increasingly efficient with $h \downarrow 0$. Recall that for this application a mat-vec requires solving the Poisson equation.

Appendix A. Some facts about the spectral distance. Throughout this section $(\mathcal{X},\langle\cdot, \cdot\rangle)$ is a real, finite dimensional Hilbert space with norm $\|\cdot\|$. All operators in this section are assumed to be in $\mathcal{L}_{+}(\mathcal{X})$ (see Section 4.2 for definition) unless otherwise specified. The following inequalities were proved in [13] (Lemma 3.2):

Lemma A.1. If $\alpha \in(0,1)$ and $z \in \mathcal{B}_{\alpha}(1)$, then

$$
\frac{\ln (1+\alpha)}{\alpha}|1-z| \leqslant|\ln z| \leqslant \frac{|\ln (1-\alpha)|}{\alpha}|1-z| .
$$

For $|\ln z| \leqslant \delta$ we have

$$
\frac{1-e^{-\delta}}{\delta}|\ln z| \leqslant|1-z| \leqslant \frac{e^{\delta}-1}{\delta}|\ln z| .
$$

Lemma A.2. Let $L, G \in \mathcal{L}_{+}(\mathcal{X})$ such that

$$
\min \left(d_{\sigma}\left(L^{-1}, G\right), d_{\sigma}\left(L, G^{-1}\right)\right) \leqslant \delta .
$$

Then

$$
\rho(I-L G) \leqslant \frac{e^{\delta}-1}{\delta} \min \left(d_{\sigma}\left(L^{-1}, G\right), d_{\sigma}\left(L, G^{-1}\right)\right)
$$

Proof. If $\lambda \in \sigma(I-L G)$ then there exists a unit vector $u \in \mathcal{X}^{\mathbb{C}}$ such that $(I-L G) u=\lambda u$, therefore

$$
(1-\lambda) u=L G u .
$$

After left-multiplying with $L^{-1}$ and taking the inner product with $u$ we obtain

$$
(1-\lambda)\left\langle L^{-1} u, u\right\rangle=\langle G u, u\rangle, \text { therefore } \lambda=1-\frac{\langle G u, u\rangle}{\left\langle L^{-1} u, u\right\rangle} .
$$


If we substitute $v=G^{-1} u$ in (A.4) and take the inner product with $v$ we have

$$
(1-\lambda) G^{-1} v=L v, \text { therefore } \lambda=1-\frac{\langle L v, v\rangle}{\left\langle G^{-1} v, v\right\rangle} .
$$

Hence, if $d_{\sigma}\left(L^{-1}, G\right) \leqslant \delta$, then

$$
\begin{aligned}
\rho(I-L G) & \leqslant \sup \left\{|1-z|: z=\langle G u, u\rangle /\left\langle L^{-1} u, u\right\rangle \text { for some } u \in \mathcal{X}^{\mathbb{C}} \backslash\{0\}\right\} \\
& \stackrel{A .2}{\leqslant} \frac{e^{\delta}-1}{\delta} d_{\sigma}\left(L^{-1}, G\right) .
\end{aligned}
$$

Instead, if $d_{\sigma}\left(L, G^{-1}\right) \leqslant \delta$, then

$$
\begin{aligned}
\rho(I-L G) & \leqslant \sup \left\{|1-z|: z=\langle L u, u\rangle /\left\langle G^{-1} u, u\right\rangle \text { for some } u \in \mathcal{X}^{\mathbb{C}} \backslash\{0\}\right\} \\
& \stackrel{\text { A.2. }}{\leqslant} \frac{e^{\delta}-1}{\delta} d_{\sigma}\left(L, G^{-1}\right) .
\end{aligned}
$$

which proves A.3.

\section{REFERENCES}

[1] V. Akçelik, G. Biros, A. Drăgănescu, O. Ghattas, J. Hill, and B. Van Bloemen WaanDERS, Inversion of airborne contaminants in a regional model, in LNCS, vol. 3993, 2006 , pp. 481-488.

[2] Volkan Akçelik, George Biros, Andrei Drăgănescu, Omar Ghattas, Judith C. Hill, and Bart G. van Bloemen WaAnders, Dynamic data driven inversion for terascale simulations: real-time indentification of airborne contaminants, in SC '05: Proceedings of the 2005 ACM/IEEE conference on Supercomputing, Washington, DC, USA, 2005, IEEE/ACM, IEEE Computer Society.

[3] Randolph E. Bank, Philip E. Gill, and Roummel F. Marcia, Interior methods for a class of elliptic variational inequalities, in Large-scale PDE-constrained optimization (Santa Fe, NM, 2001), vol. 30 of Lect. Notes Comput. Sci. Eng., Springer, Berlin, 2003, pp. 218-235.

[4] Michele Benzi, Eldad Haber, and Lauren Taralli, Multilevel algorithms for large-scale interior point methods, SIAM J. Sci. Comput., 31 (2009), pp. 4152-4175.

[5] George Biros and Günay DoĞan, A multilevel algorithm for inverse problems with elliptic PDE contraints, Inverse Problems, 24 (2008), pp. 034010, 18.

[6] Alfio Borzi AND R. GRIEsse, Experiences with a space-time multigrid method for the optimal control of a chemical turbulence model, Internat. J. Numer. Methods Fluids, 47 (2005), pp. 879-885.

[7] A. Borzì And K. Kunisch, A multigrid scheme for elliptic constrained optimal control problems, Comput. Optim. Appl., 31 (2005), pp. 309-333.

[8] Alfio Borzi And Volker Schulz, Multigrid methods for PDE optimization, SIAM Rev., 51 (2009), pp. 361-395.

[9] Susanne C. Brenner and L. Ridgway Scott, The mathematical theory of finite element methods, vol. 15 of Texts in Applied Mathematics, Springer, New York, third ed., 2008.

[10] William L. Briggs, Van Emden Henson, and Steve F. McCormick, A multigrid tutorial, Society for Industrial and Applied Mathematics (SIAM), Philadelphia, PA, second ed., 2000.

[11] ANDREI DRĂGĂNESCU, Multigrid preconditioning of linear systems for semismooth Newton methods. in preparation.

[12] — Two investigations in numerical analysis: monotonicity preserving finite element methods, and multigrid methods for inverse parabolic problems, $\mathrm{PhD}$ thesis, University of Chicago, August 2004.

[13] Andrei DrĂGănescu And Todd F. Dupont, Optimal order multilevel preconditioners for regularized ill-posed problems, Math. Comp., 77 (2008), pp. 2001-2038.

[14] Howard C. Elman, David J. Silvester, and Andrew J. Wathen, Finite elements and fast iterative solvers: with applications in incompressible fluid dynamics, Numerical Mathematics and Scientific Computation, Oxford University Press, New York, 2005. 
[15] Heinz W. Engl, Martin Hanke, and Andreas Neubauer, Regularization of inverse problems, vol. 375 of Mathematics and its Applications, Kluwer Academic Publishers Group, Dordrecht, 1996.

[16] E. M. Gertz And S. J. Wright, Object-oriented software for quadratic programming, ACM Trans. Math. Softw., 29 (2003), pp. 58-81.

[17] Philip E. Gill, Walter Murray, Dulce B. Ponceleón, and Michael A. Saunders, Primaldual methods for linear programming, Math. Programming, 70 (1995), pp. 251-277.

[18] WolfGang HackBusch, On the fast solving of parabolic boundary control problems, SIAM J. Control Optim., 17 (1979), pp. 231-244.

[19] W. Hackbusch, Fast solution of elliptic control problems, J. Optim. Theory Appl., 31 (1980), pp. $565-581$.

[20] Wolfgang HackBusch, Die schnelle Auflösung der Fredholmschen Integralgleichung, Beiträge zur Numerischen Mathematik, 9 (1981), pp. 47-62.

[21] - Multigrid methods and applications, vol. 4 of Springer Series in Computational Mathematics, Springer-Verlag, Berlin, 1985.

[22] - Integral equations, vol. 120 of International Series of Numerical Mathematics, Birkhäuser Verlag, Basel, 1995. Theory and numerical treatment, Translated and revised by the author from the 1989 German original.

[23] Martin Hanke and Curtis R. Vogel, Two-level preconditioners for regularized inverse problems. I. Theory, Numer. Math., 83 (1999), pp. 385-402.

[24] M. Hintermüller, K. Ito, And K. Kunisch, The primal-dual active set strategy as a semismooth Newton method, SIAM J. Optim., 13 (2002), pp. 865-888 (electronic) (2003).

[25] Michael Hintermüller and Michael Ulbrich, A mesh-independence result for semismooth Newton methods, Math. Program., 101 (2004), pp. 151-184.

[26] M. Hinze, R. Pinnau, M. Ulbrich, and S. Ulbrich, Optimization with PDE constraints, vol. 23 of Mathematical Modelling: Theory and Applications, Springer, New York, 2009. Edited by M. Hinze, R. Pinnau, M. Ulbrich and S. Ulbrich.

[27] Barbara Kaltenbacher, V-cycle convergence of some multigrid methods for ill-posed problems, Math. Comp., 72 (2003), pp. 1711-1730 (electronic).

[28] J. Thomas King, Multilevel algorithms for ill-posed problems, Numer. Math., 61 (1992), pp. 311-334.

[29] O. Lass, M. Vallejos, A. Borzi, and C. C. Douglas, Implementation and analysis of multigrid schemes with finite elements for elliptic optimal control problems, Computing, 84 (2009), pp. 27-48.

[30] Mitchell Luskin and Rolf Rannacher, On the smoothing property of the Crank-Nicolson scheme, Applicable Anal., 14 (1982/83), pp. 117-135.

[31] S. Menrotra, On the implementation of a primal-dual interior point method, SIAM Journal on Optimization, 2 (1992), pp. 575-601.

[32] Jorge Nocedal and Stephen J. Wright, Numerical Optimization (2nd Edition), Springer Series in Operations Research, Springer Verlag, 2006.

[33] Andreas Rieder, A wavelet multilevel method for ill-posed problems stabilized by Tikhonov regularization, Numer. Math., 75 (1997), pp. 501-522.

[34] Bert W. Rust and Dianne P. O'Leary, Confidence intervals for discrete approximations to ill-posed problems, J. Comput. Graph. Statist., 3 (1994), pp. 67-96.

[35] A. H. Stroud, Approximate calculation of multiple integrals, Prentice-Hall Inc., Englewood Cliffs, N.J., 1971. Prentice-Hall Series in Automatic Computation.

[36] Vidar Thomée, Galerkin finite element methods for parabolic problems, vol. 25 of Springer Series in Computational Mathematics, Springer-Verlag, Berlin, second ed., 2006.

[37] Michael Ulbrich, Semismooth Newton methods for operator equations in function spaces, SIAM J. Optim., 13 (2002), pp. 805-842 (electronic) (2003).

[38] Michael Ulbrich and Stefan Ulbrich, Superlinear convergence of affine-scaling interiorpoint Newton methods for infinite-dimensional nonlinear problems with pointwise bounds, SIAM J. Control Optim., 38 (2000), pp. 1938-1984 (electronic).

[39] - Primal-dual interior-point methods for PDE-constrained optimization, Math. Program., 117 (2009), pp. 435-485.

[40] M. VAllejos AND A. Bonzì, Multigrid optimization methods for linear and bilinear elliptic optimal control problems, Computing, 82 (2008), pp. 31-52.

[41] Martin Weiser, Interior point methods in function space, SIAM J. Control Optim., 44 (2005), pp. 1766-1786 (electronic). 


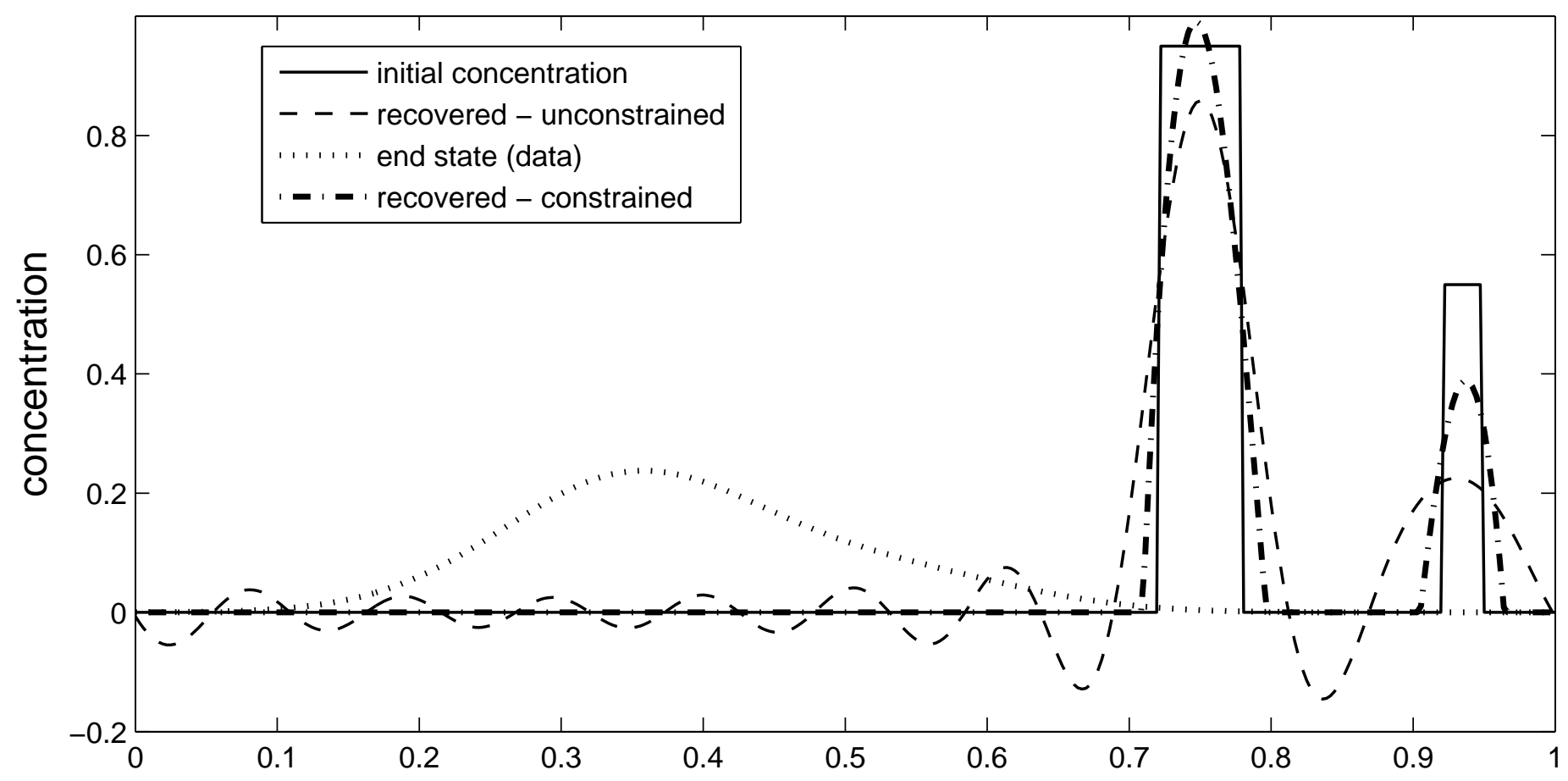




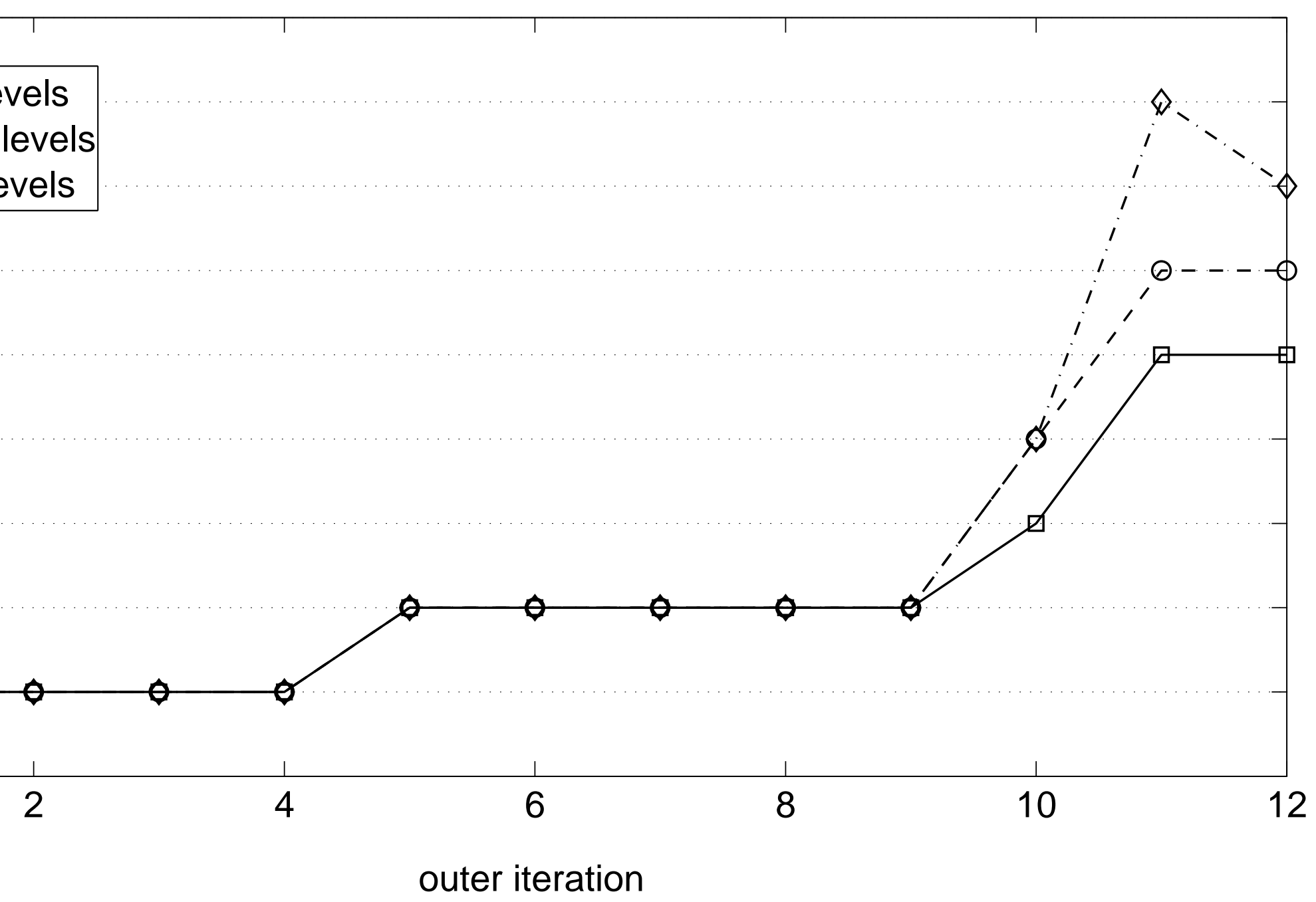

